\title{
Exploration of the rotational power consumption of a rigid flapping wing
}

\author{
Michael Truppel · Lionel Rossi
}

Received: date / Accepted: date

\begin{abstract}
The development of Micro Air Vehicles with flapping wings is inspired from the observation and study of natural flyers such as insects and birds. This article explores the rotational power consumption of a flapping wing using a mechanical flapper at $R e \simeq 4500$. This mechanical flapper is simplified to a $2 \mathrm{D}$ translation and a rotation in a water tank. Moreover, the wing kinematics are reduced to a linear translation and a rotation for the purpose of our study. We introduce the notion of non-ideal flapper and associated non-ideal rotational power. Such non-ideal devices are defined as consuming power for adding and removing mechanical power to the flow. First we use a traditional symmetrical wing kinematic which is a simplified kinematic inspired from natural flyers. The lift coefficient of this flapping is about $C_{L} \simeq 1.5$. This symmetrical wing kinematic is chosen as a reference. Further wing kinematics with asymmetric rotations are then compared to this one. These new kinematics are built using a differential velocity defined according to the translational kinematics, a time lag and a distance, $r_{k p}$. The analogy of this distance is discussed as a key point to follow along the chord. First, the wing kinematics are varied keeping a similar shape for the profiles of the angular velocity. It is shown that when compared to the reference wing kinematic, a $10 \%$ reduction of the rotational power is obtained whilst the lift is reduced by $9 \%$. Second, we release the limitation to a similar shape for the profiles of the angular velocity leading to a novel shape for the angular velocity profile named here as "double bump" profile. With these new wing kinematics, we show that a $60 \%$ reduction of the non-ideal rotational power can be achieved whilst the lift coefficient is only reduced by $1.7 \%$. Such "double bump kinematics" could then be of interest to increase the endurance of Micro-Air-Vehicles.
\end{abstract}

Keywords flapping wing $\cdot$ Lift · Power

L. Rossi

Department of Aeronautics, Imperial College London

Tel.: +44-0-20-75945047

E-mail: 1.rossi@imperial.ac.uk or rossi_lionel@yahoo.fr 


\section{Introduction}

In his "Great flight diagram", Tennekes [1997], Tennekes shows that flyers follow general laws relating to their cruise speed at sea level, $V_{\text {cruise }}$, to their weight, $W$, and wing loading, $W / S$, respectively $V_{\text {cruise }}^{2} \sim W / S$ and $V_{\text {cruise }}^{6} \sim W$. These laws concern an extremely large range of sizes, as they apply to small insects such as the crane fly, small birds such as the house wren and large aircraft such as the boeing 747. This is verified, even though current aircrafts fly with fixed wings and natural flyers use flapping wings. Nevertheless, the distribution of small flyers' characteristics presents a significant dispersion in the "Great flight diagram". Indeed, there is a large variety of flying species using flapping wings with significant variations in wing design and number, weight, size and wing kinematics. It is generally assumed that species have naturally evolved and developed performances which are vital for their survival. Consequently, the species with great maneuverability and/or flight endurance are deeply studied by scientists, e.g. dragonfly, hummingbird, bat, fruitfly, Thomas et al. [2004], Warrick et al. [2005], Tian et al. [2006], Dickinson et al. [1999]. Such natural flyers are a source of inspiration for the development of flying robots such as Micro Air Vehicle (MAV).

Since Ellington seminal work, Ellington [1984], many research have confirmed the importance and variety of unsteady mechanisms amenable to generate a lift sufficient to explain why insects can fly. A few review articles and books, e.g. Shyy et al. [2008], Wang [2005], Sane [2003], Ho et al. [2003], Azuma [2006], Shyy et al. [2010] give a nice description of the aerodynamics of small flyers. Observing that, each natural flyer has its own characteristics, the authors follow a complementary approach to the standard observation and reproduction in laboratory and numerical simulations of specific natural flyers. To do so, the wing is simplified to a rigid elliptical wing and the kinematics of the flapping are simplified to a simple translation with a rotation at the end of each stroke. This gives a reduced number of parameters to support experimental studies.

Maneuverability, lift and energy performances are three important characteristics for the development of MAV. In this paper, the authors explore the flapping performances of a mechanical wing and in particular the reduction of the rotational power while keeping significant values of lift coefficient.

Power and energy requirements are important for both natural flyers and MAV. Indeed, numerous articles and books are addressing energy and power consumption of natural flyers, e.g. Azuma [2006], Casey [1981], Dickinson et al. [1998], Hedenström and Alerstam [1995], Lehmann [2002], Pennycuick et al. [2000], Tennekes [1997], Wakeling and Ellington [1997]. The investigations of the power requirements using mechanical robots and/or numerical simulations have been the subject of recent studies. For example, recent numerical simulations have highlighted: the importance of wing kinematics and the theoretical possibility to use the flow to help the rotation of the wing, Bergou et al. [2007], the possibility to optimise the wing kinematics to minimize the energy consumption, Berman and Wang [2007], Pesavento and Wang [2009] and increase the aerodynamic performance, Bos et al. [2008], the importance of wing deformations, Young et al. [2009] and the importance of wing interaction for dragonfly, Wang and Russell [2007]. Experimental works have recently used robots to investigate power issues. For example, in their study of the hawk moth, Manduca Sexta, Zhao and Deng [2009] have found a negative work from the aerodynamic forces which helps pitching dur- 
ing stroke reversal. Lehmann [2009], Usherwood and Lehmann [2008] have shown the importance of the phasing of the dragonfly wings to improve the aerodynamic efficiency.

In this paper, the authors aim to experimentally show that the rotational power of a $2 \mathrm{D}$ mechanical flapper can be significantly reduced by varying the wing kinematics so as to capture the flow momentum to help the wing rotation. This in the same spirit as the "passive wing rotation" of natural flyers, e.g. Bergou et al. [2007]. In addition of being a new experimental investigation, this paper also makes the distinction between ideal and non-ideal flappers which should be important for the management of wing rotation for MAV. First, the authors briefly describe the experimental apparatus and measurement methods before presenting few definitions and characterising the performance of symmetric kinematics later chosen as a reference. Then the rotational kinematics of the wing are changed with a progressive increase in their complexity while the translational kinematics of the flapper are kept the same for all experiments.

\section{Description of the experimental apparatus and measurement methods}

The experimental apparatus consists in an experimental flapper with a wing immersed in water. To estimate the lift, drag and mechanical power consumption, force measurements are performed and synchronised with the wing displacements. To illustrate the flow around the wing, Particle Image Velocimetry (PIV) measurements are performed. These main experimental features are detailed hereafter.

The experimental flapper is setup within a water tank used in previous experimental works Owen et al. [2000], Owen [2001]. The working dimensions have been reduced to $760 \mathrm{mmx} 1700 \mathrm{~mm}$ for the horizontal dimensions and $350 \mathrm{~mm}$ for the vertical length. A photo of the rig with the flapper, wing and force sensor is given in Fig. 1 along with simplified schematics. The mechanical flapper is built with two translations and one rotation as indicated in Fig. 1a. To ensure smooth and accurate displacements of the wing, three servo-motors with optical encoders are coupled to three belt systems which transfer the power/motion with a gear reduction. One count of the encoders giving the motors' position corresponds to $\pi / 1000 \mathrm{rad}$. The conversion count to $\mathrm{mm}$ is about 33.37 count $/ \mathrm{mm}$ for the main translation, 32.44 count $/ \mathrm{mm}$ for the heave and 704.5 count $/ \mathrm{rad}$ for the wing rotation. Moreover, to ensure the quality of the wing kinematics, with a local feedback independent of the main computer, three servo-controllers (Agile MicroMax R) command the motors. The real position of the motors is recorded at $83,3 \mathrm{~Hz}$ and is used to characterise the wing kinematics for data processing.

The wing is mounted on the experimental flapper as illustrated in Fig. 1. The wing chord, c, is chosen as the reference length-scale. Its value is $c=54 \mathrm{~mm}$. The shape of the wing is simplified to a symmetrical ellipse and is constant along the wing span. The maximum thickness of the wing is $5 \mathrm{~mm}$, i.e. $0.0926 \mathrm{c}$, at middle chord. The wing is made of solid ABS (designed with rapid prototyping) with a $2 \mathrm{mmx} 15 \mathrm{~mm}$ thick carbon-fiber spine crossing the whole span. Outside the wing, the carbon-fiber thickness is increased to $6 \mathrm{~mm}$ (by gluing 2 additional plates) to ensure the rigidity between the wing and the force sensor. The wing is placed at $1 \mathrm{~cm}$ from the bottom wall after checking that the force measurements do not vary for distances between $0.5 \mathrm{~cm}$ and $1.5 \mathrm{~cm}$. The wet span is $29 \mathrm{~cm}$ and the wet 
area is $S \simeq 157 \mathrm{~cm}^{2}$. Among the flying insects, the position of the pitching axis is thought to lie between $0.25 \mathrm{c}$ and $0.5 \mathrm{c}$, Ellington [1984]. According to Yates [1986] the optimal position of the pitching axis is in the upper half of the wing chord and should be close to $0.25 \mathrm{c}$. Also, Dickinson [1994] has shown the importance of this pivot position. To get the effect of the asymmetrical position of the centre of rotation, we choose to connect the pitching (rotation) axis at $\mathrm{c} / 3$ from the leading edge of the wing. In previous literature, various pivot positions have been used, e.g. c/4 Kurtulus et al. [2008], Ol et al. [2008], c/3 Dickinson et al. [1999], c/2 Ansari et al. [2009].

The force measurements are performed with a force sensor mounted between the wing root and the rotational shaft, as illustrated in Fig. 1. The force sensor is an ATI Nano 17 SI-12-0.12. Its practical resolution is estimated to lie between $10 \mathrm{mN}$ and $50 \mathrm{mN}$ leading to a noise/level ratio smaller than $10 \%$ for the range of forces considered here. Force and torque calibrations are performed by varying the force intensity and direction, including positive and negative torques. We found a typical accuracy of $10 \%$ for the force measurement and $7 \%$ for the torque measurement. Moreover, the reproduction of experiments, at different dates, shows variations of the means values, e.g. lift coeffficient, of about $2 \%$ which is well below most of the variations reported in this paper due to the changes in the wing kinematics. The force sensor is reinitialised before each experimental run to remove bias and temporal variation of the reading. This is done by subtracting the mean force recording over 10s (flow at rest) one minute before the start of the motion. Moreover, at the end of the flapping, a second recording is performed with the wing at rest in order to check that this value remains unchanged after the experimental run. The force measurements performed just before and after the flapping show forces and torques fluctuations due to the residual motions in the tank (e.g. waves $)$ of about $F_{\text {wave }} \simeq 0.002 \mathrm{~N}\left(F_{\text {wave }}^{*}=3.710^{-2}\right)$ and $T_{\text {wave }} \simeq 0.01610^{-3} \mathrm{~N}$ $\left(T_{\text {wave }}^{*}=5.410^{-3}\right)$. This is two order of magnitude smaller than the force and torque intensities measured during the flapping. The measurements of the three force and torque components are performed at a very high frequency $(10 \mathrm{kHz}) \mathrm{be}-$ fore being down-sampled at $200 \mathrm{hz}$ using a temporal averaging. The force results presented in this paper have a reduced level of noise. To do so, the data is smoothed using a $3^{\text {rd }}$ order Savitzky-Golay moving average filter with a span of 65 points, i.e. $5.4 \%$ of the flapping period.

The synchronisation of the wing kinematics (i.e. motor positions) and the force measurement is important to estimate accurately the mechanical power. This is performed using the CPU clock of the main computer so as to get a temporal uncertainty smaller than $1 \mathrm{~ms}$ which is sufficient for a force and position sampling respectively at $200 \mathrm{~Hz}$ and $83 \mathrm{~Hz}$.

Flow visualisations are performed using PIV measurements. The PIV system consists of a double frame camera (Imager Pro Plus, 2048x2048 pixels, 14bit, 14Hz) coupled with a double pulse laser (Nd:Yag, New Wave Solo200XT, 532nm, 15Hz). The camera/laser control and frame recording are performed using the LAVISION camera system of the Aeronautics Department used in previous works Rossi [2010], Ferrari and Rossi [2008]. The camera is placed horizontally underneath the tank and aims at a $45^{\circ}$ mirror to see the flow. The PIV measurements are taken at middle span using a thin laser-light sheet perpendicular to the wing along with a fluorescent seeding and an optical filter. The seeding is made of fluorescent pigments: PF-38 Tropical sunlight Orange. The diameter of a pigment is about $5 \mu \mathrm{m}$. 
The 540nm cut-off optical filter removes the laser wavelength and its reflection on the black wing while the orange light from the pigments reaches the camera. The measurement domain is about $4 \mathrm{c}$ and is centred at a rotation point to prevent an optical effect between the bottom of the wing and the measurement plane. The PIV processing is performed using an in-house, e.g. Rossi et al. [2009], Rossi [2010], code based on an iterative method to adapt the size of the correlation windows with sub-pixel interpolation (and accuracy) for the estimation of the final displacement. The frames resolution is about 12.35 pixels $/ \mathrm{mm}$. The smallest correlation window is $16 \mathrm{x} 16$ pixels, i.e. $\simeq 0.024 \mathrm{c}$. The PIV are computed along a $240 \mathrm{x} 240 \mathrm{grid}$ with $50 \%$ overlap. The spacing of the velocity point on the grid is of 8 pixels, i.e. $\simeq 0.012 \mathrm{c}$.

\section{Definitions and physical parameters}

The authors now briefly present the typical parameters of their experiments in terms of Reynolds number, similitudes and differences with natural flyers, lift, drag, power and wing inertia.

The typical Reynolds number $\left(R e=\frac{u_{r e f} c}{\nu}\right)$ based on the wing chord, $c$, the maximum translational velocity, $u_{r e f} \simeq 83.5 \mathrm{~mm} / \mathrm{s}$, and the kinematic viscosity, $\nu$, is $R e \simeq 4500$. This is in the upper range of the Reynolds number envisaged for MAV, i.e 10 to 10000 .

A few similitudes and differences with natural flyers can be briefly discussed. The flapper is not a root-flapping flapper and the wing motion is $2 \mathrm{D}$. The wing's shape has been simplified to an elliptical shape which is different from a NACA0012 airfoil, e.g. Kurtulus et al. [2008] and real-shaped wings previously used with robotic experiments and numerical simulations, e.g. Dickinson et al. [1999], Usherwood and Ellington [2002], Sun and Tang [2002a]. Also, the ratio of the wing's thickness to its chord is about (9\%). This is finer than a NACA0012 and typically one order of magnitude larger than the ones of real flyers, e.g. Ellington [1984]. According to Okamoto et al. [1996] this should reduce the aerodynamic performance of the elliptical wing. We consider these kinematics as hovering because there is no incoming flow. The wing is coming back inside the flow it has induced in the previous flappings. Nevertheless, being 2D, these kinematics are different from the hovering of real flyers.

We investigate the capture of the flow (e.g. added mass) to help the rotation of the wing in water experiments. It is of interest to consider how such effect should be present in the air. We consider that the action of the pressure forces is the driving term to capture flow momentum and neglect the action of viscous forces in this matter. To compensate the ratio of densities and produce a similar dynamic pressure $\left(\frac{1}{2} \rho u^{2}\right)$ in the air, the wing should flap faster in air than water. This would imply a flapping speed of about $1.7 \mathrm{~m} / \mathrm{s}$ in air. If we compare to the flapping velocity of insects and birds in hovering flight, Azuma [2006], it is interesting to note that such a velocity magnitude corresponds to the range of few species, e.g. long-eared bat $(1.14 \mathrm{~m} / \mathrm{s})$, hover fly $(1.54 \mathrm{~m} / \mathrm{s})$, wren $(1.63 \mathrm{~m} / \mathrm{s})$, hummingbird $(1.67 \mathrm{~m} / \mathrm{s})$, bumblebee $(2.41 \mathrm{~m} / \mathrm{s})$, pigeon $(2.52 \mathrm{~m} / \mathrm{s})$.

The lift and drag coefficients are defined as follows: 


$$
C_{L}=\frac{\text { Lift }}{\frac{1}{2} \rho u_{r e f}^{2} S} ; C_{D}=\frac{\text { Drag }}{\frac{1}{2} \rho u_{r e f}^{2} S}
$$

where $u_{\text {ref }}$ is the speed of the pivot point during the purely translational motion and $\rho$ is the density of the fluid.

To measure the mechanical power, the speed of the centre of rotation, $\dot{x}_{c r}$, and the angular velocity of the wing, $\dot{\alpha}$, are computed using temporal derivations of the synchronised positions $\left(x_{c r}\right.$ and $\left.\alpha\right)$. The mechanical power can then be estimated accurately.

The mechanical power consumption related to the translations, i.e. translational power noted $P_{\text {trans }}$, is given by the scalar product of the force applied to the wing, $\mathbf{F}$, by the wing translational velocity at the centre of rotation, $\dot{\mathbf{x}}_{\mathbf{c r}}$ :

$$
P_{\text {trans }}=\mathbf{F} \cdot \dot{\mathbf{x}}_{\mathbf{c r}}
$$

The mechanical power consumption related to the rotation, i.e. rotational power noter $P_{\text {rot }}$, is given by the product of the torque applied to the wing, T, by the rotational speed of the wing:

$$
P_{\text {rot }}=T \dot{\alpha}
$$

In the present experiments, the translational kinematic is kept constant while the rotational kinematics are varied. $\frac{1}{2} \rho u_{r e f}^{3} S$ is then chosen as the power scale reference, see Tab. 1. Consequently,

$$
P_{\text {rot }}^{*}=\frac{T \dot{\alpha}}{\frac{1}{2} \rho u_{r e f}^{3} S} ; P_{\text {trans }}^{*}=\frac{\mathbf{F} \cdot \dot{\mathbf{x}}_{\mathbf{c r}}}{\frac{1}{2} \rho u_{r e f}^{3} S}
$$

The positive and negative signs indicate the interaction between the wing and the flow: positive values indicate that the wing is withdrawing energy from the flow and negative values indicate that the wing is adding energy to the flow to ensure the prescribed kinematics.

An ideal flapper would be amenable to use the energy withdrawn from the flow to support its action when it needs to add energy to the flow. In this case, the power consumption of the flapper is equal to the total mechanical power transmitted to the flow by the mechanical flapper which is the sum of the translational and rotational power:

$$
P_{\text {ideal }}=P_{\text {trans }}+P_{\text {rot }}
$$

Our mechanical flapper is controlled by three independent servo motors ensuring that the prescribed kinematics are followed. It does not have the possibility to "release its muscles" so as to let the flow move the wing or the possibility to store energy when the mechanical power is positive. We then consider the power consumption of a non-ideal flapper. This non-ideal flapper is not able to use the energy that it withdraws from the flow when the power is positive. Moreover, it needs to work to remove this energy from the flow. In this latter case of nonideal flapper, the total power consumption is the sum of the absolute values of translational and rotational powers: 


$$
P_{\text {non-ideal }}=\left|P_{\text {trans }}\right|+\left|P_{\text {rot }}\right| .
$$

We then estimate the average translational and rotational powers of a non-ideal flapper as:

$$
\left\langle\left|P_{\text {rot }}^{*}\right|\right\rangle=\frac{1}{T} \int_{0}^{T}\left|P_{\text {rot }}^{*}\right| d t ;\left\langle\left|P_{\text {trans }}^{*}\right|\right\rangle=\frac{1}{T} \int_{0}^{T}\left|P_{\text {trans }}^{*}\right| d t
$$

The effects of the wing inertia have been quantified by performing the wing kinematics in air whilst the final experiments are performed in water. It is found that the wing inertia has a negligible contribution to lift and drag. The force, torque and power measured are one order of magnitude smaller in air than in water. Consequently, the main part of the forces measured in water are due to the hydrodynamic forces. Also, we do not remove the wing inertia from our water measurements.

\section{Standard symmetrical wing kinematic}

First, a reference flapping is studied and quantified in terms of lift, drag and power. This reference flapping is inspired from simplified symmetrical kinematics used in previous literature, e.g. Dickinson et al. [1999]. Moreover, the wing kinematics of this reference flapping are simplified to a translation and a rotation at the end of each stroke. Fig. 2 gives the position of the elliptical wing with a time step equals to $\mathrm{T} / 20$ where $\mathrm{T}$ is the period of the flapping. The reference (or standard) wing kinematics ensure the continuity of the position, velocity and also acceleration to prevent any jerk of the wing. Their equations over half a period are respectively given in equations 8 and 9 for translation and rotation.

The translation consists in a displacement with a constant speed at mid stroke; followed by a sinusoidal deceleration/acceleration of the wing at the end of each stroke. The characteristics of the translational velocities are: the reference (and maximal) velocity of the wing during pure translation, $u_{r e f}=83.46 \mathrm{~mm} / \mathrm{s}$, the root mean square velocity over one period, $u_{r m s}=72.5 \mathrm{~mm} / \mathrm{s}$, the mean velocity over one period $u_{\text {mean }}=66.67 \mathrm{~mm} / \mathrm{s}$. In all experiments the wing translates for 3.7 chord length, i.e. $20 \mathrm{~cm}, T=6 s, t_{1}=-0.1 T, t_{0}=0, t_{2_{t}}=0.1 T$.

$$
\begin{aligned}
-\frac{T}{4}<t \leq t_{1} \dot{x}_{c r} & =u_{r e f} \\
t_{1}<t \leq t_{0} \dot{x}_{c r} & =0.5 u_{r e f}\left(1+\cos \left(\pi \frac{t-t_{1}}{t_{0}-t_{1}}\right)\right) \\
t_{0}<t \leq t_{2 t} \dot{x}_{c r} & =-0.5 u_{r e f}\left(1-\cos \left(\pi \frac{t-t_{2 t}}{t_{2_{t}}-t_{0}}\right)\right) \\
t_{2_{t}}<t \leq \frac{T}{4} \dot{x}_{c r} & =u_{r e f}
\end{aligned}
$$

The standard symmetrical rotation of the wing is performed using a sinusoidal kinematic at the end of each stroke. In all experiments, $\alpha_{1}=\frac{\pi}{4}, \alpha_{2}=\frac{3 \pi}{4}$. In the case of the symmetrical rotation, $t_{2}=t_{2_{t}}=0.1 T$. In section 5 , asymmetric kinematics are used and $t_{2}$ varies whilst $t_{2}$ is kept constant. 


$$
\begin{aligned}
-\frac{T}{4} & <t \leq t_{1} \dot{\alpha}=0 \\
t_{1} & <t \leq t_{0} \dot{\alpha}=\frac{\alpha_{2}-\alpha_{1}}{t_{2}-t_{1}}\left(1-\cos \left(2 \pi \frac{t-t_{1}}{t_{2}-t_{1}}\right)\right) \\
t_{0} & <t \leq t_{2} \dot{\alpha}=\frac{\alpha_{2}-\alpha_{1}}{t_{2}-t_{1}}\left(1-\cos \left(2 \pi \frac{t-t_{1}}{t_{2}-t_{1}}\right)\right) \\
t_{2} & <t \leq \frac{T}{4} \dot{\alpha}=0
\end{aligned}
$$

For clarity, only one part of the flapping $(-T / 4<t<T / 4)$ including two regions of translation at constant speed and one rotation is detailed. The kinematics of the other half-period are symmetric. Fig. 3a gives the translational and rotational speeds respectively normalised by $u_{r e f}$ and $\dot{\alpha}_{r e f}=2 u_{r e f} / c$ where $\mathrm{c}$ is the wing chord. The plotted values are extracted from the temporal derivation of the recorded positions of the wing. Fig. $3 \mathrm{~b}$ gives the position of the centre of rotation normalised by the wing chord, i.e. the wing's displacement $x / c$, and the smallest geometrical angle of the wing respectively to the translational direction, $\theta$, often referred as the "angle of attack of the wing". Recorded values are close to the theoretical position, i.e. within few $\%$ of the reference scale. ${ }^{1}$

This flapping generates a net (average) lift coefficient of about $\left\langle C_{L}\right\rangle=1.49$. The typical temporal evolution of the instantaneous lift coefficient, $C_{L}(t)$, is given in Fig. 4. Dotted lines indicate the times when the wing translational and rotational kinematics vary according to equations 9 and 8 . The deceleration of the wing corresponds to a drop of the lift which starts to raise again when the wing accelerates. Moreover, the lift produced is positive during the main part of the flapping which is in agreement with previous numerical simulations and experiments, e.g. Birch et al. [2004], Dickinson and Götz [1993], Dickinson et al. [1999], Kurtulus et al. [2005], Sane and Dickinson [2001], Sun and Tang [2002b,a], Wang et al. [2004]. We may indicate here that the use of a fully sinusoidal kinematic for the translation while conserving the same amplitude and frequency, e.g. Lehmann and Pick [2007], leads to an increase of $4.5 \%$ in lift whilst the power consumption increases by $9.6 \%$ when compared to the chosen standard kinematic.

In the present experiments, the wing rotation starts after the deceleration. We do not observe a significant peak in the lift curve related to the start of rotation as it can be the case for kinematics with a rotation starting before the wing deceleration, e.g. Dickinson et al. [1999], Kurtulus et al. [2005], Sane and Dickinson [2001]. Whilst this does not prevent the flapper to produce significant lift coefficients, this is a noticeable difference between our simplified flapper and real flyers, or robots, with 3D wing's motion, e.g. Dickinson et al. [1999], Sane and Dickinson [2001]. Significant values of the lift coefficient are observed at the start of translation, i.e. right after the change of direction, and during the translation at constant speed. The lift at the start of the wing's translation corresponds to an impulse lift, as previously observed e.g. Dickinson and Götz [1993] with typical values of lift coefficient higher than 2. The main lift is observed when the wing moves at a constant speed and angle between the wing and the translation direction. Numerous studies have previously discussed that high values of lift coefficient can be attributed to the presence of a leading edge vortex, e.g. Bomphrey et al.

\footnotetext{
1 The main position errors are due to the mechanical flapper inertia. They appear like a delay (in time) between theoretical positions and the recorded ones. They are about $3 \%$ of the chord for $x$ and half a degree for $\alpha$. If the delay is bypassed, the same profiles are observed and the differences between theoritical and recorded velocitites are within $1 \%$ of the reference scales.
} 
[2006, 2005], Birch and M.H. [2001], Birch et al. [2004], Ellington et al. [1996], van der Berg and C.P. [1997], Muijres et al. [2008]. Fig. 5a gives a PIV velocity field measured at the end of the pure translation stage, i.e. just before the start of the wing rotation. The streamlines show the presence of a large vortex attached to the wing. ${ }^{2}$ Fig. $5 \mathrm{~b}$ gives the vorticity field in the trailing edge region. The two maxima of vorticity correspond to small vortical structures shedded from the trailing edge. The time between these two shedding can be estimated using the vertical distance between the two vortical structures and the velocity of the wing. This time is about $0.073 \mathrm{~T}$.

Fig. 4 gives also the temporal evolution of the drag coefficient over an entire stroke starting at the middle of the translation. The drag curves evolve similarly to the lift curve, with comparable intensities if we except the highest drag peak during the acceleration stage. Moreover, the period of the oscillations observed on lift and drag coefficients is in agreement with the time estimated between two vortex shedding. As illustrated in Fig. 2, the wing is moving forward and backward on a linear path with a wing rotation at both extremities. The drag changes direction according to the wing motion and the "horizontal" force compensates on both half-strokes. In the absence of mean thrust, we consider that the mechanical wing is hovering.

Few differences can be noticed between the two halves of the flapping. They are mainly due to a modification of the angle of attack and of the flow around the wing between the two half-strokes. Fig. 6 shows a velocity field at the start of the constant speed and constant angle regime. The presence of a flow induced by the flapping wing normally to the translational direction can be observed. The typical velocity intensity of this flow is about $0.4 u_{\text {ref }}$. Such induced velocity reduces the effective angle of attack by more than $20^{\circ}$. This flow fluctuates in time and is not perfectly orthogonal to the wing displacement. In addition, a small incertitude (less than a degree) in the wing angle zero reference can contribute to these differences. Nevertheless, Tab. 2 shows that these differences do not significantly impact the mean values computed over the entire period and each half, with variations within few $\%$.

The rotational power consumption is the main focus of this paper. Fig. 7 gives the dimensionless rotational power $P_{\text {rot }}^{*}$ and the torque $T_{z}^{*}$ during a flapping period. During the rotation stage, the wing is first adding energy to the flow, $P_{\text {rot }}^{*}<0$, before withdrawing energy from the flow, $P_{\text {rot }}^{*}>0$. Moreover, the presence of peak in rotational power is related to peak values of the torque.

The average non-ideal rotational power, $\left\langle\left|P_{\text {rot }}^{*}\right|\right\rangle$, is about 0.09 with small variations of about $2 \%$ between the first and second half periods, see Tab 2. The average ideal rotational power, $\left\langle P_{r o t}^{*}\right\rangle$ is significantly weaker than the non-ideal rotational power with values of about 0.016 and -0.007 during the first and second half periods respectively. If the mechanical flapper was ideal and able to transfer energy between the two rotations, its average rotational power would be twenty times weaker than its non-ideal rotational power.

\footnotetext{
2 One curiosity of this vortex is the presence of more than one core within the main recirculation. Such feature has been previously observed in numerical simulations and experiments, e.g. Srygley and Thomas [2002], Lu and Shen [2008], Lu et al. [2007, 2006]. The existence of a multi-core vortex or possibly a mis-shapen single core vortex is not necessarily relevant to the purposes of this paper.
} 
It is important to note that the average non-ideal rotational power is about $30 \%$ of the average translational power during the rotation of the wing and about $7 \%$ of the average translational power during the entire flapping.

We will now explore how the flow can be used to reduce the rotational power consumption of non-ideal flappers or MAV.

\section{Variations of the wing kinematics}

The wing kinematics are now varied to capture momentum from the flow to help to turn the wing. Only the rotational speed is varied, the translation being kept the same for all experiments. ${ }^{3}$ It is expected that this will reduce the rotational power consumption similarly to the passive wing rotation in insect flights e.g. Bergou et al. [2007]. First, the flapping kinematics are kept similar to the standard symmetrical wing kinematic. Such kinematics are named "single bump" as they refer to a single "acceleration-deceleration" for the rotation of the wing. Second, the discussion is extended to improve the wing kinematics so as to reduce the rotational power consumption. These new kinematics are named "double bump" as they refer to a double "acceleration-deceleration" for the rotation of the wing.

5.1 Variation of the wing kinematics: single bump

To mathematically link the wing rotation to its translation, a simplified description is introduced. This is equivalent to define the wing rotation as:

$$
\dot{\alpha}=\frac{u_{\text {flow }} \sin \alpha}{r_{k p}}
$$

where $u_{\text {flow }}$ is a differential velocity between the velocity of the flow and the velocity of the centre of rotation due to the deceleration and acceleration of the wing at the end of each stroke and $r_{k p}$ is a length-scale parameter. The differential velocity is implemented using the difference between translational velocities at time $t$ and $t-\Delta t$ :

$$
u_{\text {flow }}=\dot{x}_{c r}(t)-\dot{x}_{c r}(t-\Delta t)
$$

This construction presents the advantage of defining $u_{\text {flow }}$ according to the wing kinematics without considering the real flow pattern. The position of the length-scale, $r_{k p}$, and the temporal lag $\Delta t$ are then the only parameters to adjust to vary the wing rotation. Before varying these two parameters, a brief and simplified analogy is used to show that the length-scale $r_{k p}$ corresponds to the position of a key point to follow along the wing chord.

\footnotetext{
$3 u_{\text {ref }}, t_{0}$ and $t_{2_{t}}$ are unchanged.
} 


\subsubsection{Simplified analogy for $r_{k p}$}

Fig. 8 illustrates two simplified cases (with and without leading edge vortex) where the flow behind the wing goes faster than the wing's centre of rotation due to the wing deceleration.

Due to the wing rotation, the wing velocity respectively to its centre of rotation is not uniform. This velocity is here noted $r \dot{\alpha}$ where $r$ is the algebraic distance from a point on the wing chord to the centre of rotation and $\dot{\alpha}$ is the angular velocity of the wing. The deceleration of the wing increases the difference between the velocity of the flow and the velocity of the centre of rotation. This differential velocity is noted $u_{\text {flow }}$. It corresponds to the velocity of the flow in the referential of the centre of rotation. A dynamic pressure term is built as, $\frac{1}{2} \rho\left(u_{\text {flow }} \sin \alpha-r \dot{\alpha}\right)^{2}$, where $\alpha$ is the angle of the wing with the direction of translation. This pressure term generates a torque, $T_{\text {flow }}$, along the wing span which is written as follows:

$$
T_{\text {flow }} \simeq \int_{\text {wing }} \frac{1}{2} \rho\left(u_{\text {flow }} \sin \alpha-r \dot{\alpha}\right)^{2} r w_{\text {span }} d r
$$

where $w_{\text {span }}$ is the wing span length.

In this simplified analogy, the point along the wing chord where the built dynamic pressure is null is $r_{k p}$, i.e. one of the two parameters to adjust in equation 10. To give a simplified description, at this point the velocity of the wing follows the flow. It follows by construction that:

$$
T_{\text {flow }} \simeq \frac{1}{2} \rho \dot{\alpha}^{2} w_{\text {span }} \int_{\text {wing }}\left(r_{k p}-r\right)^{2} r d r
$$

The positions $r_{k p}$ can now be chosen to minimize $\left|\int_{w i n g}\left(r_{k p}-r\right)^{2} r d r\right|$. According to cases a and b given in Fig. 8, two key positions can be defined. They are respectively given in equations 14 and 15 where the origin of the $\mathrm{r}$ axis is the centre of rotation of the wing.

$$
\begin{aligned}
& \min \left(\left|\int_{-c / 3}^{2 c / 3}\left(r_{k p}-r\right)^{2} r d r\right|\right) \leadsto r_{k p} \simeq 0.22 c \\
& \min \left(\left|\int_{0}^{2 c / 3}\left(r_{k p}-r\right)^{2} r d r\right|\right) \leadsto r_{k p} \simeq 0.44 c
\end{aligned}
$$

These two positions are significantly different. This shows the importance of the flow pattern to estimate the relevant positions of $r_{k p}$ and the limit of this simplified description.

\subsubsection{Wing kinematics}

A new set of asymmetric kinematics is defined for the rotation, the translation being unchanged. They are given in equation 16, where the time $t_{2}$ is computed so as to reach the desired value of $\alpha$ at the end of the wing rotation. 


$$
\begin{aligned}
-\frac{T}{4}<t & \leq t_{1} \dot{\alpha}=0 \\
t_{1} & <t \leq t_{0} \dot{\alpha}=\left(\dot{x}_{c r}(t)-\dot{x}_{c r}(t-\Delta t)\right) r_{k p}^{-1} \sin \alpha \\
t_{0} & <t \leq t_{2} \dot{\alpha}=\dot{\alpha}\left(t_{0}\right) \cos ^{2}\left(\frac{\pi}{2} \frac{t-t_{0}}{t_{2}-t_{0}}\right) \\
t_{2} & <t \leq \frac{T}{4} \dot{\alpha}=0
\end{aligned}
$$

$t_{1}$ is the time when the wing starts to decelerate. $t_{0}$ is the time when the translational speed changes direction. If $\Delta t=t-t_{1}$ then $u_{\text {flow }}=\dot{x}_{c r}(t)-u_{\text {ref }}$. In other cases $\Delta t$ is kept constant. The reference velocity is then used to evaluate $u_{\text {flow }}$ when $t \leq t_{1}+\Delta t$. Then, when $t \geq t_{1}+\Delta t$ a deceleration of the flow is introduced according to the past translational velocity.

The angular velocity of the wing is now varied in experiments, according to equation 10 .

\subsubsection{Variation of the rotational power with $r_{k p}$}

First, to explore the variations of the power consumption with $r_{k p}, u_{f l o w}$ is set to $u_{\text {flow }}=\dot{x}_{c r}(t)-u_{\text {ref }}$ and $r_{k p}$ is varied.

Fig. 9 gives the profiles of the angular velocity, $\dot{\alpha}^{*}=\dot{\alpha} / \dot{\alpha}_{r e f}=c \dot{\alpha} / 2 u_{r e f}$, and geometrical angle, $\theta$ of the wing for different $r_{k p}$. The profiles corresponding to the symmetrical rotation is added in Fig. 9 for comparison. When $r_{k p}=0.309 c$ the wing turns faster than in the case of the standard rotation and does so for a shorter time. When $r_{k p}=0.849 c$, the wing turns slower than in the case of the standard rotation and does so for a longer time. When $r_{k p}=0.54 c$ the profile of the angular velocity of the wing is close to one of the standard rotation with two noticeable differences: the wing turns faster and its kinematic is asymmetric with more rotation taking place before the change of translational direction.

Fig. 10 gives the rotational power $P_{\text {rot }}^{*}$ and the torque $T_{z}^{*}$ during half a period for the wing kinematics illustrated in Fig. 9. The profiles of $P_{\text {rot }}^{*}$ of these kinematics are now compared to the one of the standard rotation. As it could be expected, if the wing turns fast (i.e. $r_{k p}=0.309 c$ ) there is a large increase of the rotational power intensity before the change of the flapping direction. If the wing turns slowly, i.e. $r_{k p}=0.849 c$, there is a change of sign of the rotational power before the change of direction, meaning that the mechanical wing needs to slow down the flow to keep the prescribed trajectory. Also, the rotational power is mainly positive during rotation and there is a significant increase of the rotational power after the change of flapping direction. When $r_{k p}=0.54 c$, the rotational power intensity seems to be reduced both before and after the change of the flapping direction.

Fig. 11 gives the quantitative values of the average non-ideal rotational power, $\left\langle\left|P_{\text {rot }}^{*}\right|\right\rangle$ and the lift coefficient, $\left\langle C_{L}\right\rangle$, while varying $r_{k p}$. The values corresponding to the standard rotation are indicated using a dash line for the rotational power and a dot and dash line for the lift coefficient. It is striking that using these new kinematics with $u_{\text {flow }}=\dot{x}_{c r}(t)-u_{\text {ref }}$ does not significantly reduce the rotational power consumption. In fact, for $r_{k p}=0.54 c$ the rotational power is reduced by $3 \%$. Also, it can be noted that the lift coefficient does not vary significantly for $r_{k p} \leq 0.6 c$.

To the authors' perspective, this shows that the standard wing kinematic, which is a simplified kinematic inspired from flying birds and insects, is already a good kinematic.

The value of $r_{k p}$ is now set to $0.54 \mathrm{c}$. 
5.1.4 Variation of the rotational power with $\dot{x}_{c r}(t-\Delta t)$

Fig. 12 gives the angular velocity, $\dot{\alpha}$, and the geometrical angle, $\theta$ of the wing for different values of $\Delta t$ during half a period. The symmetrical rotation is added to Fig. 12 for comparison. The values of $\Delta t$ are given in percent of $\mathrm{T}$ and the duration of the translational deceleration is $10 \%$ of $\mathrm{T}$. When $\Delta t=7.5 \%, u_{\text {flow }}=$ $\dot{x}_{c r}(t)-u_{r e f}$ during most of the wing deceleration. When $\Delta t=4.2 \%$, the wing rotation is quickly decelerated when compared to the symmetrical case. In this case, $\theta$ reaches $90^{\circ}$ after the change of the flapping direction.

Fig. 13 gives the rotational power and the lift coefficient during half a period for the kinematics illustrated in Fig. 12. Both long and short $\Delta t$ lead to an increase in rotational power intensities. The intermediate value, $\Delta t=0.058 T$ seems to slightly reduce the rotational power intensity when compared to the standard symmetrical rotation.

Fig. 14 gives the average rotational power, $\left\langle\left|P_{\text {rot }}^{*}\right|\right\rangle$, and lift coefficient, $\left\langle C_{L}\right\rangle$, for different values of $\Delta t$ while keeping $r_{k p}=0.54 \mathrm{c}$. It appears that for values of $\Delta t$ between $0.05 \mathrm{~T}$ and $0.07 \mathrm{~T}$ the rotational power is reduced when compared to the standard symmetrical rotation. For $\Delta t=0.058 T$ the rotational power is reduced by about $10 \%$. Nevertheless, this reduction in power consumption comes with a loss of lift performance of about $9 \%$.

These results show that the power consumption can be slightly reduced using asymmetric "single bump" kinematic for the angular velocity. Furthermore, this confirms that the standard symmetrical rotation is a good kinematic.

The authors now explore wing kinematics with a double bump for the angular velocity.

5.2 New "double bump" wing kinematics

\subsubsection{Wing kinematics}

After the deceleration stage, the translational velocity changes direction and the wing accelerates. During this stage, it is the flow velocity in front of the wing which can be used to reduce the torque. To use the flow to help the wing rotate, the differential velocity $\left(\dot{x}_{c r}(t)-\dot{x}_{c r}(t-\Delta t)\right)$ is now extended beyond the point of change of direction, i.e. beyond $t_{0}$. With $\delta t_{2}=0.05 T, t_{2}$ is computed to ensure that the wing reaches the desired angle at the end of the rotation.

$$
\begin{aligned}
-\frac{T}{4}<t \leq t_{1} \quad \dot{\alpha} & =0 \\
t_{1}<t \leq t_{2}-\delta_{t_{2}} & \dot{\alpha}=\left(\dot{x}_{c r}(t)-\dot{x}_{c r}(t-\Delta t)\right) r_{k p}^{-1} \sin \alpha \\
t_{2}-\delta_{t_{2}}<t \leq t_{2} \quad \dot{\alpha} & =\dot{\alpha}\left(t_{2}-\delta_{t_{2}}\right) \cos ^{2}\left(\frac{\pi}{2} \frac{t-t_{2}+\delta_{t_{2}}}{\delta_{t_{2}}}\right) \\
t_{2}<t \leq \frac{T}{4} \quad \dot{\alpha} & =0
\end{aligned}
$$

When the wing changes direction, the additional velocity due to the wing acceleration makes the flow in front of the wing tend towards a configuration with a uniform flow velocity, similar to Fig 8a. This may be seen as moving the critical point to follow closer to the centre of rotation. Consequently, the wing rotation should likely be increased beyond the one obtained by conservation of the previous 
key point velocity. This new acceleration of the wing rotation is introduced using the following kinematics:

$$
\begin{array}{cl}
-\frac{T}{4}<t \leq t_{1} & \dot{\alpha}=0 \\
t_{1}<t \leq t_{0} & \dot{\alpha}=\left(\dot{x}_{c r}(t)-\dot{x}_{c r}(t-\Delta t)\right) r_{k p}^{-1} \sin \alpha \\
t_{0}<t \leq t_{0}+\delta_{t_{0}} & \dot{\alpha}=\dot{\alpha}\left(t_{0}\right)+\int_{t_{0}}^{t} \ddot{\alpha}\left(t_{0}\right) \cos \left(\frac{\pi}{2} \frac{t-t_{0}}{\delta_{t_{0}}}\right) d t \\
t_{0}+\delta_{t_{0}}<t \leq t_{2}-\delta_{t_{2}} & \dot{\alpha}=\dot{\alpha}\left(t_{0}+\delta_{t_{0}}\right)+p \frac{2 u_{r e f}}{c} * \sin ^{2}\left(\frac{\pi}{2} \frac{t-t_{0}-\delta_{t_{0}}}{t_{2}-\delta_{t_{2}}-t_{0}-\delta_{t_{0}}}\right) \\
t_{2}-\delta_{t_{2}}<t \leq t_{2} & \dot{\alpha}=\dot{\alpha}\left(t_{2}-\delta_{t_{2}}\right) \cos ^{2}\left(\frac{\pi}{2} \frac{t-t_{2}+\delta_{t_{2}}}{\delta_{t_{2}}}\right) \\
t_{2}<t \leq \frac{T}{4} & \dot{\alpha}=0
\end{array}
$$

with $\delta t_{0}=0.021 T, \delta t_{2} \simeq 0.01 T$

Fig. 15 illustrates the experimental values of the kinematics defined in equations (17), (18) and (9). The natural double bump kinematic, given by equation 17 , is noted rkp while the tuned kinematic, given by equation 18 , is noted 0.621 according to the dimensionless value of $p$ coefficient, $p=0.621$. The main differences between the natural double bump kinematic and the tuned one are a deeper (longer) deceleration followed by a higher acceleration reaching a higher maximum of angular velocity close to the end of the wing rotation.

Fig. 16 gives the temporal evolution of the rotational power during half a period for the kinematics illustrated in Fig. 15. The use of a double bump kinematic (noted rkp) dramatically reduces the large peak observed during the wing acceleration for the standard symmetrical rotation. It can be noted here that a faster variation of $\theta$ after it reaches $90^{\circ}$ is also observed for the fruit fly, e.g. Bos et al. [2008].

When compared to the symmetrical rotation, the natural double bump kinematic reduces the rotational power by about $46 \%$ while the lift coeficient is only reduced by $5.5 \%$. Moreover, the rotational power is further reduced by the tuning of the double bump wing kinematic, as illustrated by the curve with $p=0.621$ in Fig. 16. Fig. 17 gives the lift coefficient for these kinematics during half a period. It can be noticed that the double bump kinematic leads to a peak of lift during the second increase (bump) in the angular velocity profile.

Fig. 18 gives the average rotational power, $\left\langle\left|P_{\text {rot }}^{*}\right|\right\rangle$, and lift coefficient, $\left\langle C_{L}\right\rangle$, versus the values of $p$. The values for the natural double bump are indicated using dashed lines and the values for the symmetrical rotation are indicated using dotted lines. The tuning of the wing's kinematics by varying $p$ shows that when compared to the symmetrical rotation, the average rotational power can be reduced by $59 \%$ while the lift coefficient is only reduced by $1.7 \%$.

Also, it can be noticed that in Fig. 15b the difference between the two double bump kinematics is not as clear as in Fig. 15a. As these two kinematics present significant variations in the rotational power and lift coefficient, this point highlights the importance of temporal derivatives when describing the wing kinematics of the mechanical flapper. It is likely that this should also be true for the wing kinematics of natural flyers and MAV.

These results show that for a mechanical flapper with a rigid wing and without the possibility to release the rotation, the use of a double bump kinematic dramatically reduces the energy costs due to rotation when compared to the simple symmetrical kinematics. 


\section{Conclusion}

The performances of a rigid flapping wing have been explored for selected wing kinematics and the notion of non-ideal flappers and rotational power has been introduced.

To start with, a standard symmetrical wing kinematic which is a simplified kinematic inspired from natural flyers has been studied. This simplified kinematic generates an unsteady lift leading to an average lift coefficient of $\left\langle C_{L}\right\rangle \simeq 1.5$. Then, the dependence of the rotational power to the wing angular velocity has been investigated. The main values obtained for the reduction of the average rotational power and lift are summarized in Tab. 3. First, the variations of the rotational kinematics are limited to keep similarity with the standard kinematic (single bump) while introducing asymmetry. They are noted as single bump in Tab. 3. With this limitation, it is found that the rotational power is reduced by $3 \%$ when compared to the flapping with a symmetrical rotation. Second, new wing kinematics are introduced to reduce the rotational power. Indeed, following a key point on the wing chord during both wing deceleration and wing acceleration, i.e. after it changes direction, leads to double bump kinematics for the angular speed. They are noted as double bump in Tab. 3. These new wing kinematics dramatically reduce the rotational power consumption by about $60 \%$ while keeping a good lift coefficient with a reduction of only $1.7 \%$ when compared to standard symmetrical wing kinematics.

This paper shows the importance of asymmetric wing rotations and kinematics derivatives to reduce the rotational power. In particular, the use of a double bump kinematic could be of interest to increase the endurance of MAV. Possible similarities with the real complexity of the wing kinematics of natural flyers, such as for example the fruit fly, Bos et al. [2008], are let for future investigations.

Acknowledgements The authors acknowledge colleagues and students who have contributed to the development of the experimental flapper: Aero's workshop and his head R. Hutchins, P. Bearman, I. Bousfield, M. Cwizewicz, A. Dorobantu, M. Ducarouge, J.M.R. Graham, A. Jasudasen, B. Labat, S. Lardeau. They also acknowledge the support of the EPSRC, grant $\mathrm{EP} / \mathrm{D} 072034 / 1$.

\section{References}

S.A. Ansari, N. Phillips, G. Stabler, P.C. Wilkins, R. Zbikowski, and Knowles K. Experimental investigation of some aspects of insect-like flapping flight aerodynamics for application to micro air vehicles. Exp. Fluids, 2009.

Akira Azuma. The biokinetics of flying and swimming. Aiaa edition, 2006.

A.J. Bergou, S. Xu, and Z.J. Wang. Passive wing pitch reversal in insect flight. J. Fluid Mech., 591:321-337, 2007.

G.J. Berman and Z.J. Wang. Energy-minimizing kinematics in hovering insect flight. J. Fluid Mech., 582:153-168, 2007.

J.M. Birch and Dickinson M.H. Spanwise flow and the attachment of the leadingedge vortex on insect wings. Nature, 412:729-733, 2001.

J.M. Birch, Dickinson W.B., and Dickinson M.H. Force production and flow structure of the leading edge vortex on flapping wings at high and low reynolds numbers. J. exp. Biol., 207:1063-1072, 2004. 
R.J. Bomphrey, N.J. Lawson, J.H. Harding, G.K. Taylor, and Thomas A.L.R. The aerodynamics of manduca sexta: digital particle image velocimetry analysis of the leading-edge vortex. J. exp. Biol., 208:1079-1094, 2005.

R.J. Bomphrey, N.J. Lawson, G.K. Taylor, and A.L.R. Thomas. Application of digital particle image velocimetry to insect aerodynamics: measurement of the leading-edge vortex and near wake of a hawkmoth. Exp. Fluids, 40:546-554, 2006.

F.M. Bos, D. Lentink, B.W. Van Oudheusden, and H. Bijl. Influence of wing kinematics on aerodynamic performance in hovering insect flight. J. Fluid Mech., 594:341-368, 2008.

T.M. Casey. A comparison of mechanical and energetic estimate of floght cost for hovering sphinx moths. J. exp. Biol., 91:117-129, 1981.

M.H. Dickinson. The effects of wing rotation on unsteady aerodynamic performance at low reynolds numbers. J. exp. Biol., 192:179-206, 1994.

M.H. Dickinson and K.G Götz. Unsteady aerodynamic performance of model wings at low reynolds numbers. J. exp. Biol., 174:45-64, 1993.

M.H. Dickinson, Lehmann F.O., and Pang Chan W. The control of mechanical power in insect flight. Amer. Zool., 38:718-728, 1998.

M.H. Dickinson, F-O Lehmann, and S.P. Sane. Wing rotation and the aerodynamic basis of insect flight. Science, 284:1954-1960, 1999.

C.P. Ellington. The aerodynamics of hovering insect flight. Philosophical Transactions of the Royal Society of London. Series B, Biological Sciences, 305(1122): 1-181, 1984.

C.P. Ellington, C. Van der Berg, A.P. Willmott, and Thomas A.L.R. Leading-edge vortices in insect flight. Nature, 384:626-630, 1996.

S Ferrari and L Rossi. "Particle tracking velocimetry and accelerometry (ptva) measurements applied to quasi-two-dimensional multi-scale flows". Exp. Fluids, 44:873-886, 2008.

A. Hedenström and T. Alerstam. Optimal flight speed of birds. Phil. Trans. R. Soc. Lond. B, 348:471-487, 1995.

S. Ho, H. Nassef, N. Pornsinsirirak, Y-C Tai, and Ho C-M. Unsteady aerodynamics and flow control for flapping wing flyers. Progress in Aerospace Sciences, 39:636$681,2003$.

D.F. Kurtulus, A. Farcy, and N. Alemdaroglu. Unsteady aerodynamics of flapping airfoil in hovering flight at low reynolds numbers. AIAA, Reno:1356, 2005.

D.F. Kurtulus, L. David, A. Farcy, and N. Alemdaroglu. Aerodynamic characteristics of flapping motion in hover. Exp Fluids, 44:23-36, 2008.

F.O. Lehmann. The constraints of body size on aerodynamics and energetics in flying fruit flies: an integrative view. Zoology, 105:287-295, 2002.

F.O. Lehmann. Wingwake interaction reduces power consumption in insect tandem wings. Exp Fluids, 46:765-775, 2009.

F.O. Lehmann and S. Pick. The aerodynamic benefit of wingwing interaction depends on stroke trajectory in flapping insect wings. The Journal of Experimental Biology, 210:1362-1377, 2007.

Y. Lu and G.X. Shen. Three-dimensional flow structures and evolution of the leading-edge vortices on an flapping wing. The Journal of Experimental Biology, 211:1221-1230, 2008.

Y. Lu, G.X. Shen, and G.J. Lai. Dual leading-edge vortices on flapping wings. The Journal of Experimental Biology, 209:5005-5016, 2006. 
Y. Lu, G.X. Shen, and W.H. Su. Flow visualisation of dragonfly hovering via an electromechanical model. AIAA Journal, 45:615-623, 2007.

F.T. Muijres, L.C. Johansson, R. Barfield, M. Wolf, Spedding G.R., and A. Hedenström. Leading-edge vortex improves lift in slow-flying bats. Science, 319:1250-1253, 2008.

M. Okamoto, K. Yasuda, and A. Azuma. Aerodynamic characteristics of the wings and body of a dragonfly. The Journal of Experimental Biology, 199:281-294, 1996.

M.V. Ol, L. Bernal, C-K Kang, and W. Shyy. Aerodynamic characteristics of flapping motion in hover. Exp Fluids, 44:23-36, 2008.

J.C. Owen. PhD thesis, Passive control of vortex shedding in the wakes of bluff bodies. University of london edition, 2001.

J.C. Owen, P.W. Bearman, and A. Szewcyzk. Suppression of karman vortex shedding. Phys. Fluids, 12(9):1-13, 2000.

C.J. Pennycuick, A. Hedenström, and M. Rosén. Horizontal flight of a swallow (hirundo rustica) observed in a wind tunnel, with a new method for directly measuring mechanical power. The Journal of Experimental Biology, 203:17551765, 2000.

U. Pesavento and Z.J. Wang. Flappingwing flight can save aerodynamic power compared to steady flight. Phys. Rev. Lett., 103:118102, 2009.

L. Rossi. A mechanism to explore lamination rate. Phys. Rev. E., 81:027301, 2010.

L. Rossi, S. Bocquet, S. Ferrari, J.M. Garcia de la Cruz, and S. Lardeau. Control of flow geometry using electromagnetic body forcing. Int J. Heat Fluid Flow, 30: 505-513, 2009.

S. P. Sane. The aerodynamics of insect flight. The Journal of Experimental Biology, 206:4191-4208, 2003.

S. P. Sane and M.H. Dickinson. The control of flight force by a flapping wing: lift and drag production. The Journal of Experimental Biology, 204:2607-2626, 2001.

W. Shyy, H. Aono, S.K. Chimakurthi, P. Trizila, C.K. Kang, C.E.S. Cesnik, and H. Liu. Recent progress in flapping wing aerodynamics and aeroelasticity. Progress in Aerospace Sciences, 46:284-327, 2010.

Wei Shyy, Yongsheng Lian, Jian Tang, Dragos Viieru, and Liu Hao. Aerodynamics of low reynolds number flyers. cambridge aerospace series edition, 2008.

R.B. Srygley and A.L.R. Thomas. Unconventional lift-generating mechanisms in free-flying butterflies. Nature, 420:660-664, 2002.

M. Sun and J. Tang. Unsteady aerodynamic force generation by a model fruit fly wing in flapping motion. The Journal of Experimental Biology, 205:55-70, 2002a.

M. Sun and J. Tang. Lift and power requirements of hovering flight in drosophila virilise. The Journal of Experimental Biology, 205:2413-2427, 2002b.

Henk Tennekes. The simple science of Flight. Mit press edition, 1997.

A.L.R. Thomas, G.K. Taylor, R.B. Srygley, R.L Nudds, and R. Bomphrey. Dragonfly flight: free-flight and tethered flow visualizations reveal a diverse array of unsteady lift-generating mechanisms, controlled primarily via angle of attack. The Journal of Experimental Biology, 207:4299-4323, 2004.

X. Tian, J. Iriarte-Diaz, K. Middleton, R. Galvao, E. Israeli, A. Roemer, A. Sullivan, A. Song, S. Swartz, and K. Breuer. Direct measurements of the kinematics and dynamics of bat flight. Bioinsp. Biomim.S10S18, 1:S10-S18, 2006.

J.R. Usherwood and C.P. Ellington. The aerodynamic of revolving wings i. model hawkmoth wings. The Journal of Experimental Biology, 205:1547-1564, 2002. 
J.R. Usherwood and F.O. Lehmann. Phasing of dragonfly wings can improve aerodynamic efficiency by removing swirl. J.R. Soc. Interface, 5:1303-1307, 2008.

C. van der Berg and Ellington C.P. The three-dimensional leading-edge vortex of a 'hovering' model hawkmoth. Phil. Trans. R. Soc. Lond. B, 352:329-340, 1997.

J.M. Wakeling and C.P. Ellington. Dragonfly flifht, iii. lift and power requirements. The Journal of Experimental Biology, 200:583-600, 1997.

Z. J. Wang. Dissecting insect flight. Annu. Rev. Fluid. Mech., 37:183-210, 2005.

Z. J. Wang and D. Russell. Effect of forewing and hindwing interactions on aerodynamic forces and power in hovering dragonfly flight. Phys. Rev. Lett., 99:148101, 2007.

Z. J. Wang, J. M. Birch, and M. H. Dickinson. Unsteady forces and flows in low reynolds number hovering flight: two-dimensional computations vs robotic wing experiments. The Journal of Experimental Biology, 207:449-460, 2004.

D.R. Warrick, B.W. Tobalske, and D.R. Powers. Aerodynamics of the hovering hummingbird. Nature, 435:1094-1097, 2005.

G.T. Yates. Optimum pitching axes in flapping wing propulsion. J. Theor. Biol., 120:255-276, 1986.

J. Young, S. M. Walker, Bomphrey R.J., Taylor G.K., and Thomas A.L.R. Details of insect wing design and deformation enhance aerodynamic function and flight efficiency. Science, 325:1549, 2009.

L. Zhao and X. Deng. Power distribution on the hovering flight of the haw moth manduca sexta. Bioinsp. Biomim., 4, 2009. 
Table 1 Reference scales

\begin{tabular}{ll}
\hline scale & reference used \\
\hline Length & $c=54 m m$ (wing chord) \\
Time & $T=6 s$ (flapping period) \\
Velocity & $u_{\text {ref }}=0.08346 \mathrm{~m} / \mathrm{s}$ \\
Angular velocity & $\dot{\alpha}_{\text {ref }}=2 u_{\text {ref }} / \mathrm{c}=3.074 \mathrm{~s}^{-1}$ \\
Lift and drag & $F_{\text {ref }}=\frac{1}{2} \rho u_{r e f}^{2} S=0.0544 N$ \\
Torque & $T_{r e f}=\frac{1}{2} \rho u_{r e f}^{2} S c=2.9410^{-3} \mathrm{Nm}$ \\
Powers & $P_{r e f}=\frac{1}{2} \rho u_{r e f}^{3} S=4.5410^{-3} W$ \\
\hline
\end{tabular}

Table 2 Dimensionless force coefficients and rotational power for the reference flapping with a symmetric rotation. $\left\langle C_{L}\right\rangle$ and $\left\langle C_{D}\right\rangle$ are the mean lift and drag coefficients. $\left\langle\left|P_{\text {rot }}^{*}\right|>\right.$ is the average non-ideal rotational power. These average values are estimated over 10 flapping from periods 10 to 19 .

\begin{tabular}{lccc}
\hline Mean over & $\left\langle C_{L}\right\rangle$ & $\left\langle C_{D}\right\rangle$ & $\left\langle\left|P_{\text {rot }}^{*}\right|\right\rangle$ \\
\hline Entire periods & 1.49 & 1.57 & 0.09 \\
Half periods (first) & 1.45 & 1.47 & 0.092 \\
Half periods (second) & 1.53 & 1.66 & 0.088 \\
\hline
\end{tabular}

Table 3 Summary of the variations obtained with asymmetric wing kinematics, when compared to the reference flapping with a standard symmetrical rotation. $\left\langle C_{L}\right\rangle$ and $\left\langle\left|P_{\text {rot }}^{*}\right|\right\rangle$ are respectively the lift coefficient, the non-ideal rotational power. These average values are estimated over 10 flapping from periods 10 to 19 .

\begin{tabular}{llc}
\hline wing kinematics & $\left\langle C_{L}\right\rangle$ & $\left\langle\left|P_{\text {rot }}^{*}\right|\right\rangle$ \\
\hline Single bump (rkp) & $+1 \%$ & $-3 \%$ \\
Single bump (rkp with time lag) & $-9 \%$ & $-10 \%$ \\
Double bump (natural) & $-5.5 \%$ & $-46 \%$ \\
Double bump (tuned) & $-1.7 \%$ & $-59 \%$ \\
\hline
\end{tabular}



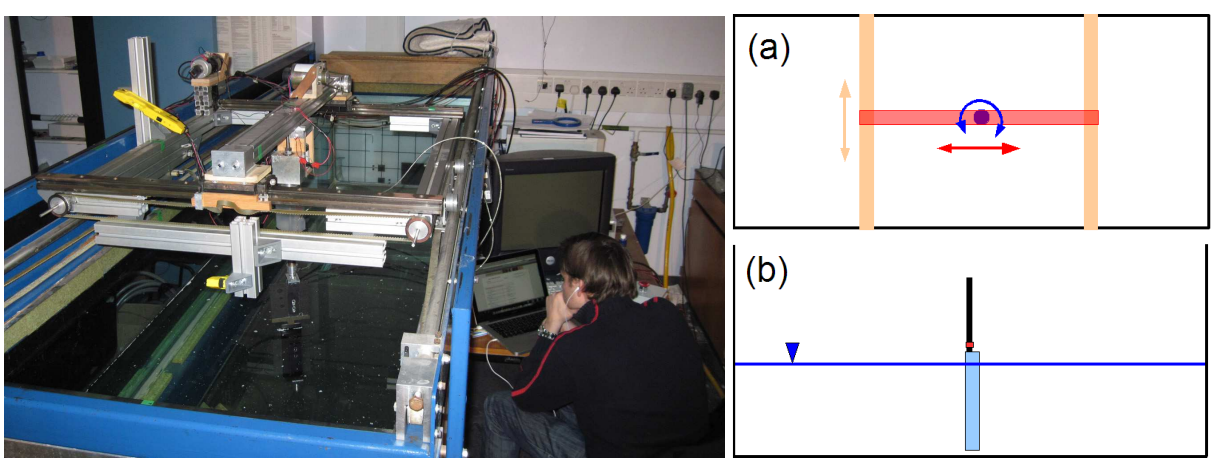

Fig. 1 Photography of the experimental rig with the flapper and simplified schematics: (a) top view indicating the displacement along the 3 -axes, i.e. two translations and one rotation,

(b) side view showing the wing immersed within water. The gap between the wing and the bottom wall is $1 \mathrm{~cm}(0.185 \mathrm{c})$ and the water depth is $30 \mathrm{~cm}(5.56 \mathrm{c})$.

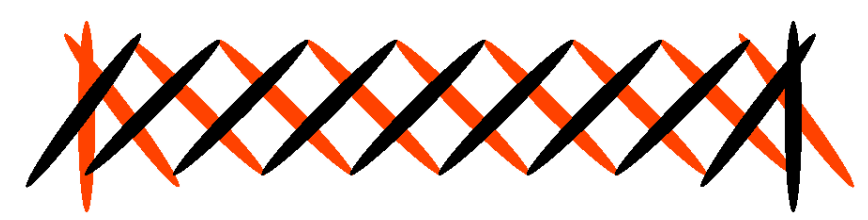

Fig. 2 Illustration of the displacement of the elliptical wing. The time spacing between two positions of the wing is $T / 20$. Black and grey (orange online) colours correspond respectively to a displacement from left to right and right to left. 


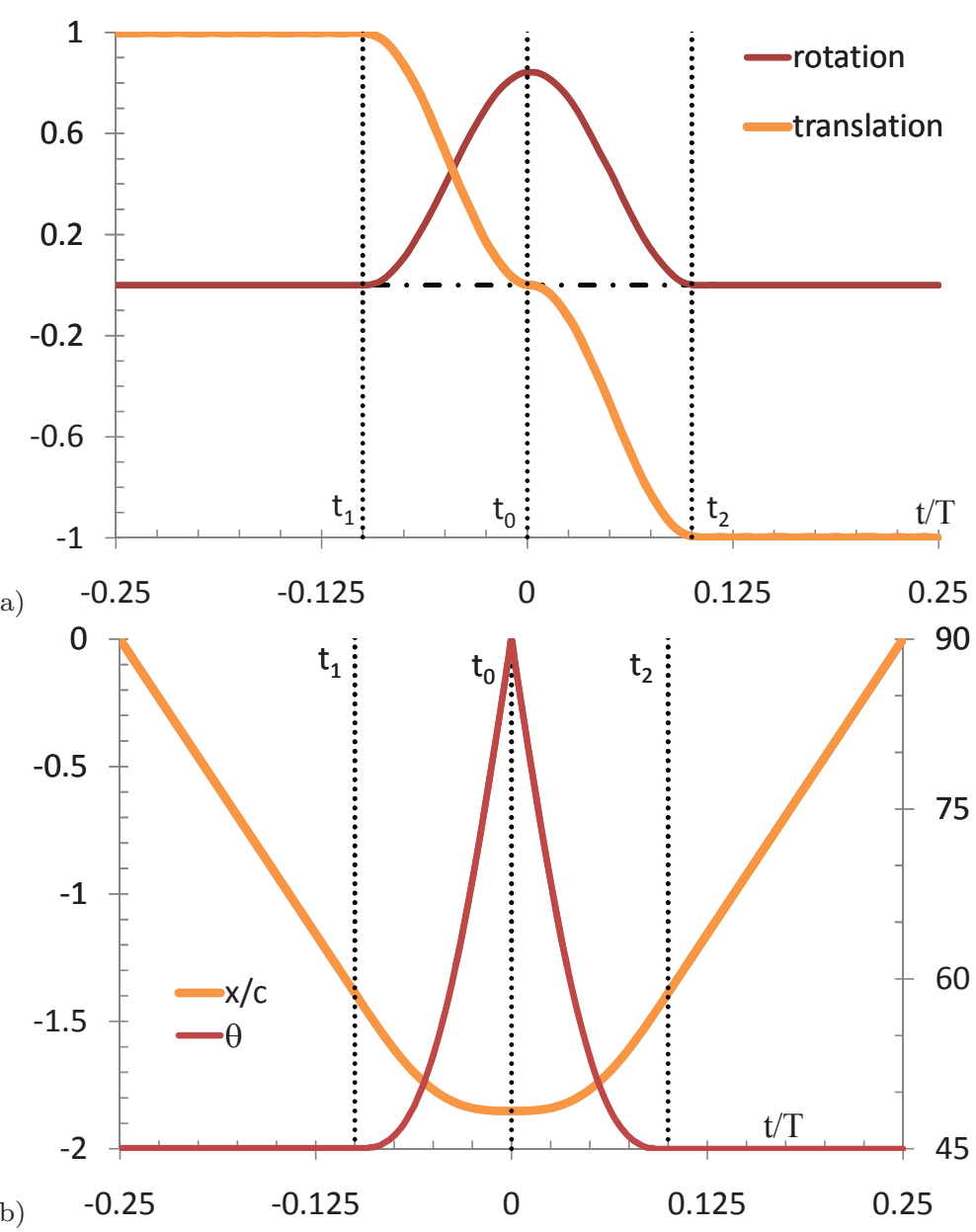

Fig. 3 (a) Dimensionless translational $\left(\dot{x} / u_{r e f}\right)$ and rotational $\left(c \dot{\alpha} / 2 u_{r e f}\right)$ speeds. These values are obtained by derivation of the recorded positions of the servo-motors. (b) Geometrical angle of the wing, $\theta$ (right vertical axis) and displacement, $x / c$ (left vertical axis). For (a)\&(b) the values are plotted during half a period for the standard kinematic with a symmetrical rotation. $\mathrm{t} / \mathrm{T}$ is plotted in abscissa. Dotted lines indicate the key times given in equations (8) and (9). 


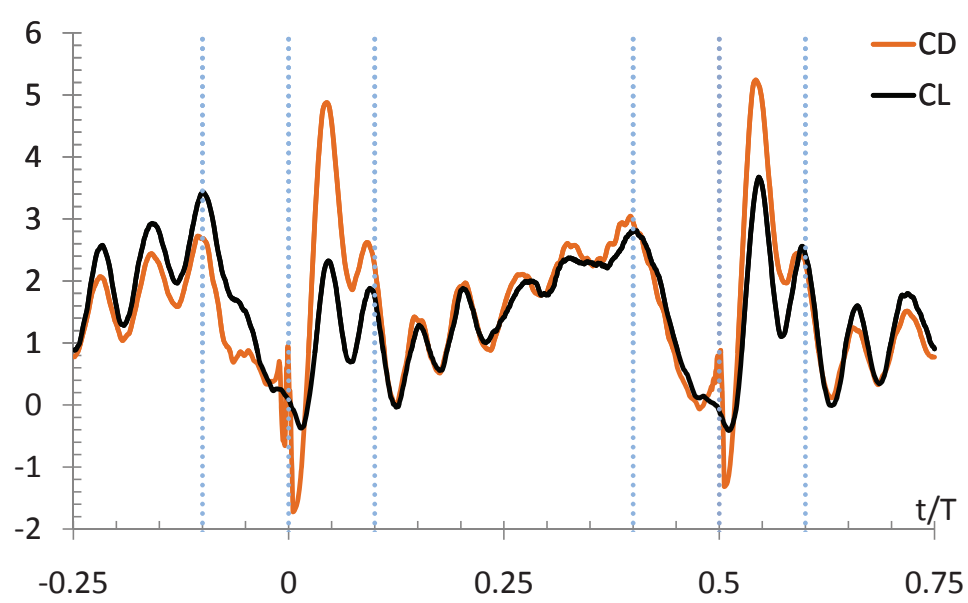

Fig. 4 Lift and Drag coefficients during one period for the reference wing kinematic with a symmetrical rotation. Phase average over periods 10 to 19. t/T is plotted in abscissa.

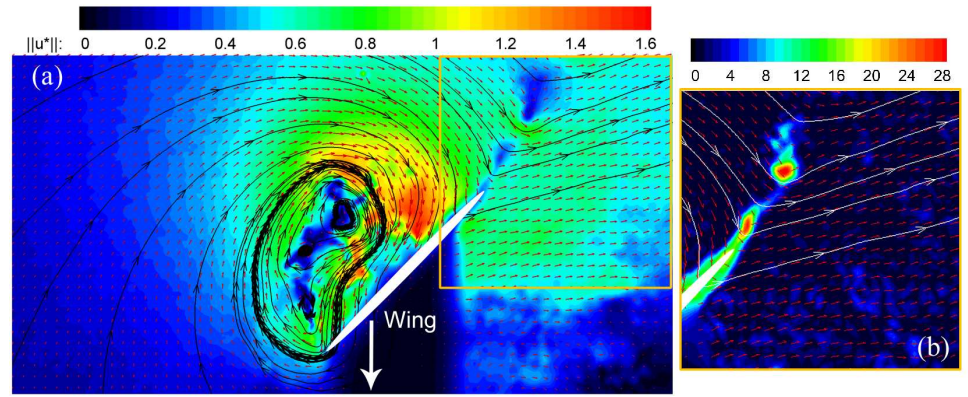

Fig. 5 Illustration of the leading edge vortex and trailing edge vortices using PIV measurements. Only $1 / 16^{t h}$ of the velocity vectors are plotted using arrows. This indicates the grid resolution and velocity direction. (a) Instantaneous velocity field. Black lines are streamlines. The velocity colormap refers to the dimensionless magnitude of velocity with $u^{*}=\mathbf{u}_{\mathbf{P I V}} / u_{\text {ref }}$. The dark region below the wing is due to the wing's shadow. (b) Vorticity field of the trailing edge wake region highlighted by an orange square in (a). The vorticity colormap refers to the dimensionless magnitude of the vorticity with $\omega^{*}=\left\|\operatorname{rot}\left(\mathbf{u}_{\mathbf{P I V}}\right)\right\| /\left(u_{r e f} / c\right)$. White lines are streamlines. 


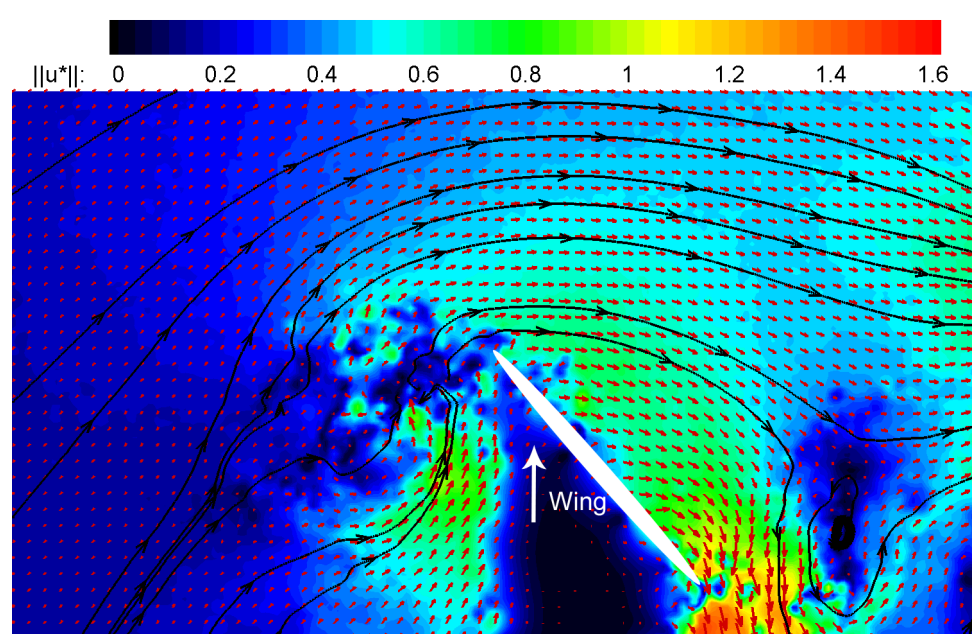

Fig. 6 Velocity field obtained by PIV at the start of the displacement of the wing with a constant angle and a constant velocity. Only $1 / 16^{\text {th }}$ of arrows are plotted to indicate the grid resolution and the velocity direction. $u^{*}=\mathbf{u}_{\mathrm{PIV}} / u_{\text {ref }}$. The dark region below the wing is due to the wing's shadow. Black lines are streamlines.

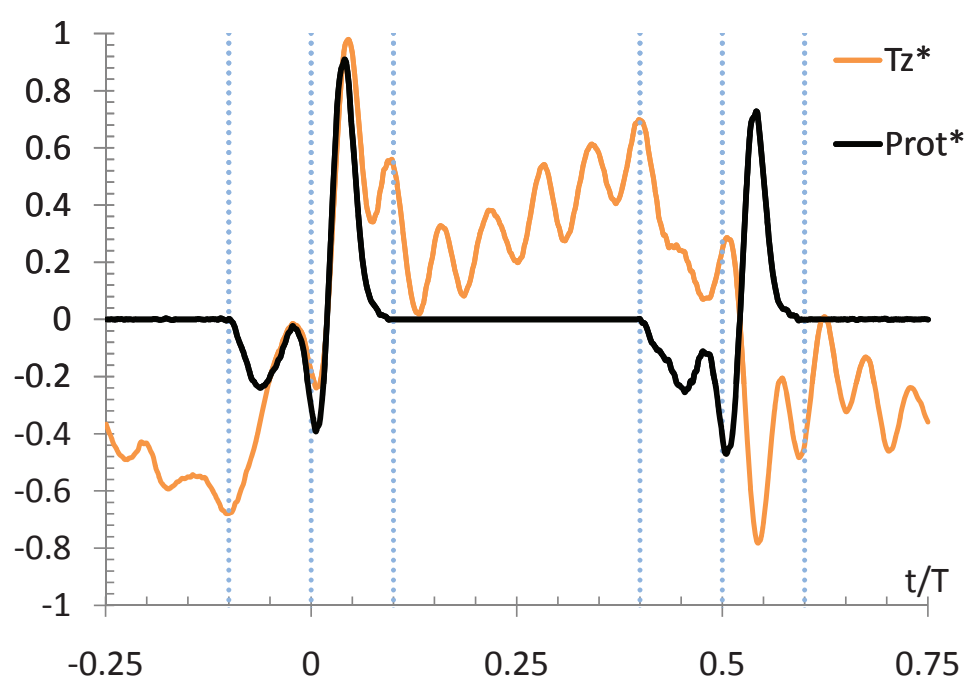

Fig. 7 Torque, $T_{z}^{*}$, and rotational power, $P_{r o t}^{*}$. Phase average over periods 10 to $19 . \mathrm{t} / \mathrm{T}$ is plotted in abscissa. 

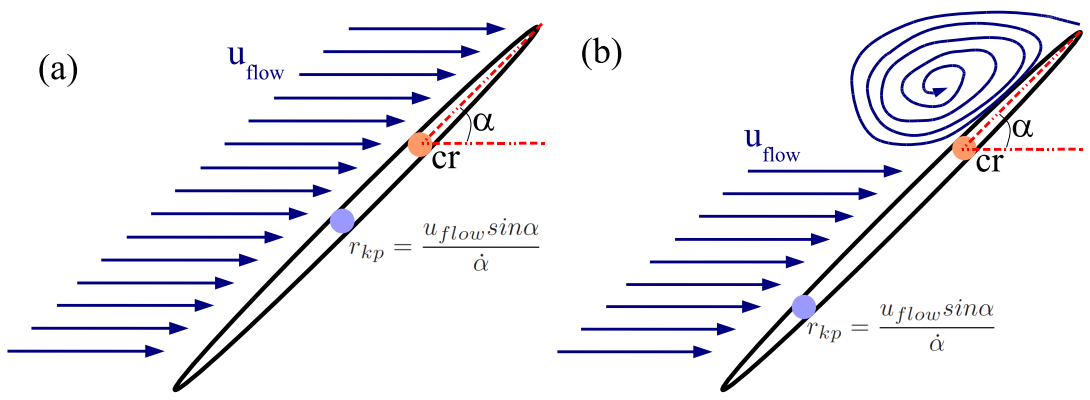

Fig. 8 Illustration of the flow behind the wing during deceleration in two simplified cases: (a) uniform velocity along the chord (b) uniform velocity on the lower part with the presence of a leading edge vortex. $u_{\text {flow }}$ is the velocity relative to the centre of rotation due to the wing deceleration, $\alpha$ is the angle of the wing with the direction of translation, $c r$ is the centre of rotation of the wing, $r_{k p}$ is a reference key point where the wing velocity in the direction of the translation equals $u_{\text {flow }}$. 

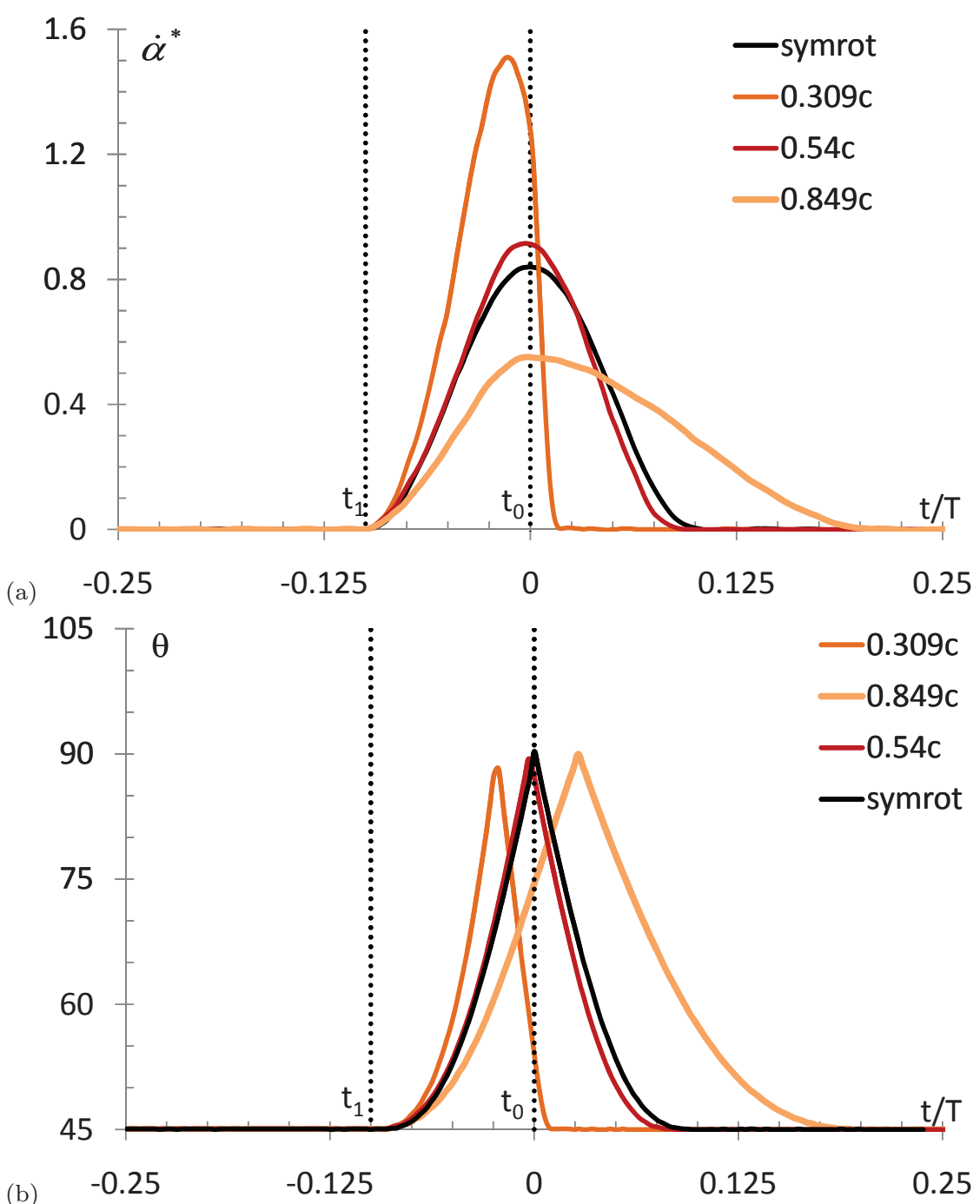

Fig. 9 (a) Dimensionless angular velocities of the wing $\dot{\alpha}^{*}=c \dot{\alpha} / 2 u_{\text {ref }}$. (b) Geometrical angle of the wing, $\theta$. For (a) \& (b) the values are plotted during half a period, for the standard symmetrical rotation, symrot, and selected values of $r_{k p}$, i.e. $0.309 \mathrm{c}, 0.54 \mathrm{c}, 0.849 \mathrm{c}$ where $\mathrm{c}$ is the wing's chord. Dotted lines at time $t_{1}$ and $t_{0}$ indicate respectively the start of the rotation and the change of the translational direction. The curves plotted are exported from recorded experimental data. 

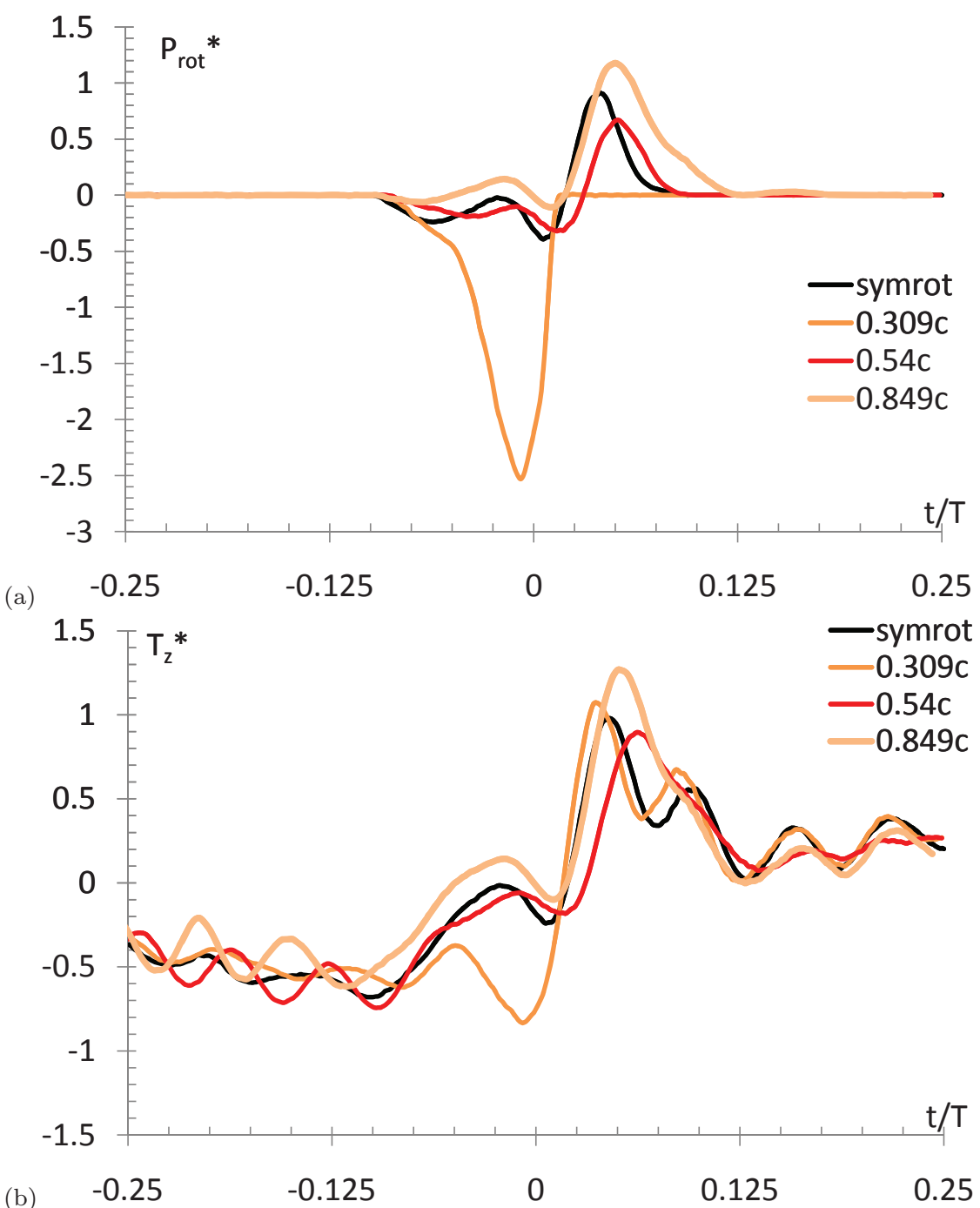

Fig. 10 Dimensionless rotational power, $P_{r o t}^{*},(\mathrm{a})$ and torque, $T_{z}^{*}$, (b) during half a period. For (a) \& (b) the values are plotted for the standard symmetrical rotation, symrot, and selected values of $r_{k p}$, i.e. $0.309 \mathrm{c}, 0.54 \mathrm{c}, 0.849 \mathrm{c}$ where $\mathrm{c}$ is the wing's chord. These results correspond to a phase average over periods 10 to $19 . \mathrm{t} / \mathrm{T}$ is plotted in abscissa. 


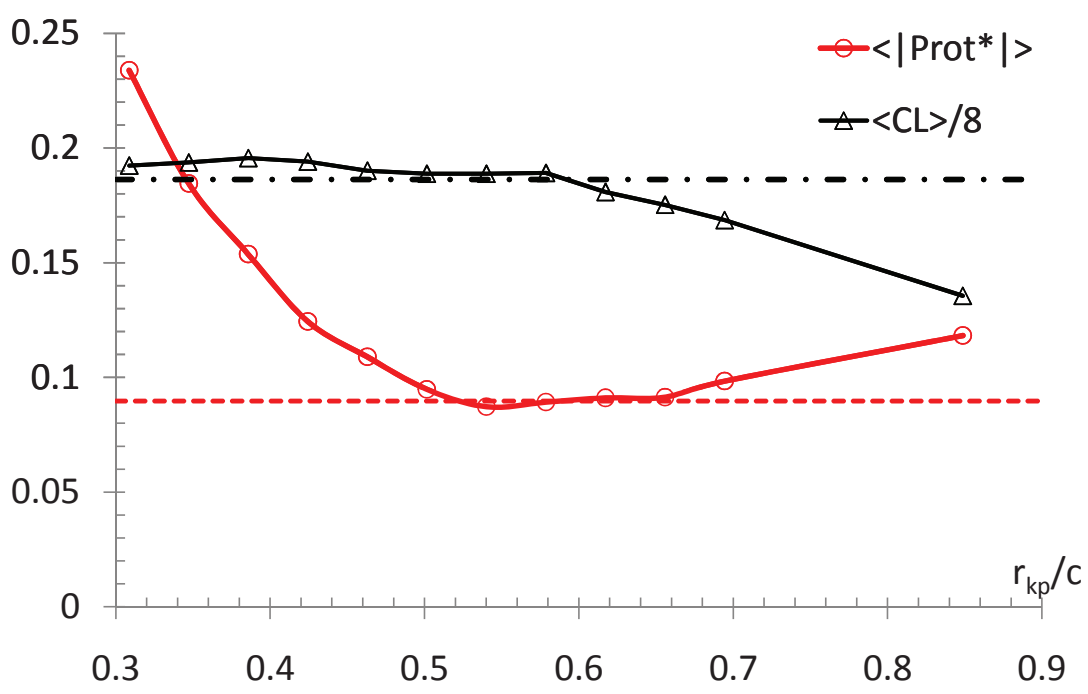

Fig. 11 Average non-ideal rotational power, $\left\langle\left|P_{\text {rot }}^{*}\right|>\right.$, and lift coefficient, $\left\langle C_{L}\right\rangle / 8$, versus $r_{k p} / c$ values given in abscissa. The dot-dash and dashed lines indicate the values obtained for the symmetrical rotation for lift and power respectively. 


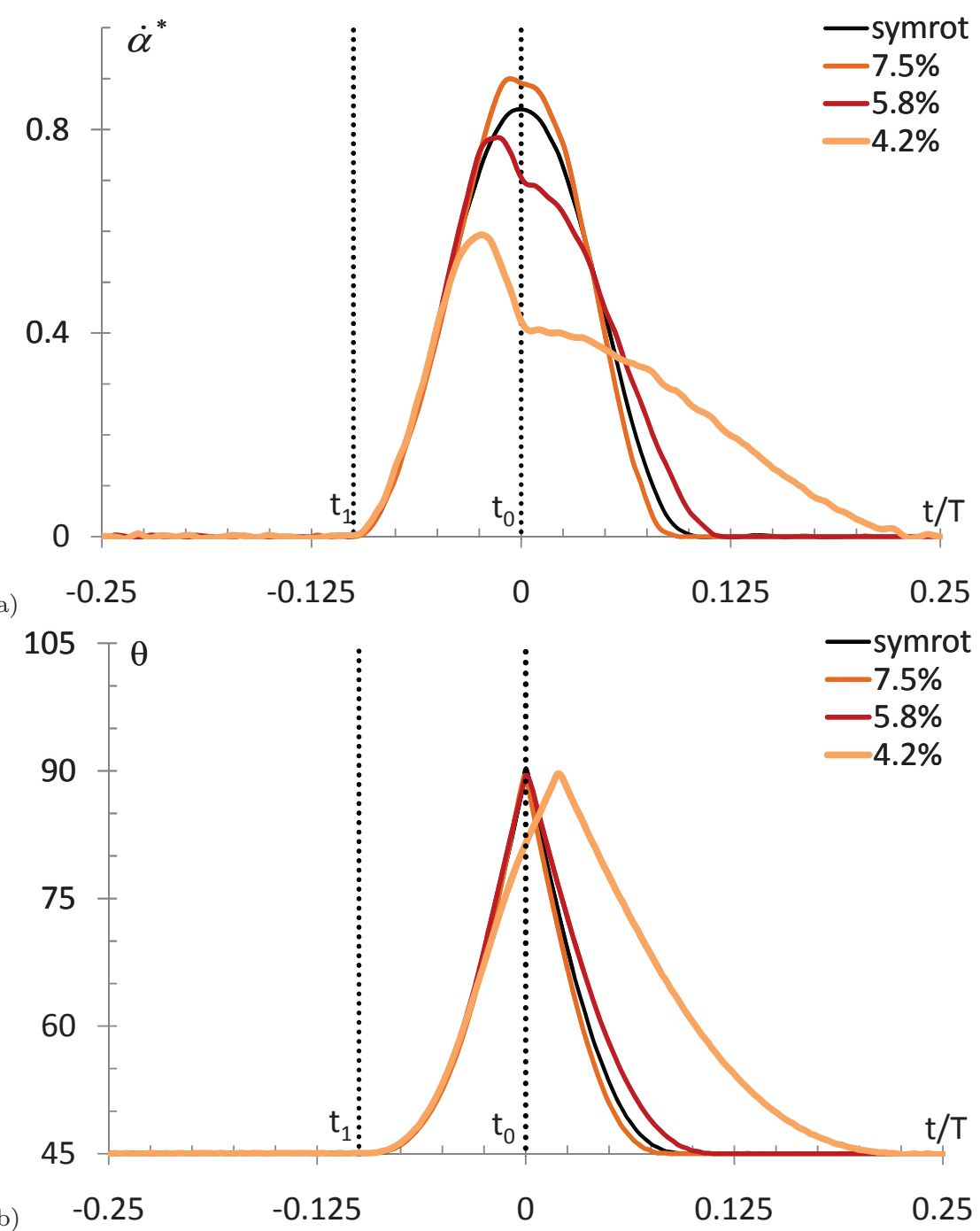

Fig. 12 Dimensionless angular velocity, $\dot{\alpha}^{*}$ (a), and geometrical angle, $\theta$ (b), of the wing. For (a) \& (b) the values are plotted during half a period, for the standard symmetrical rotation, symrot, and selected values of $\Delta t$, i.e. $0.075 \mathrm{~T}, 0.058 \mathrm{~T}, 0.042 \mathrm{~T}$; with $r_{k p}=0.54 c$. The vertical dotted lines at $t_{1}$ and $t_{0}$ indicate respectively the start of the rotation and the change of the translational direction. The curves plotted are exported from recorded experimental data. 

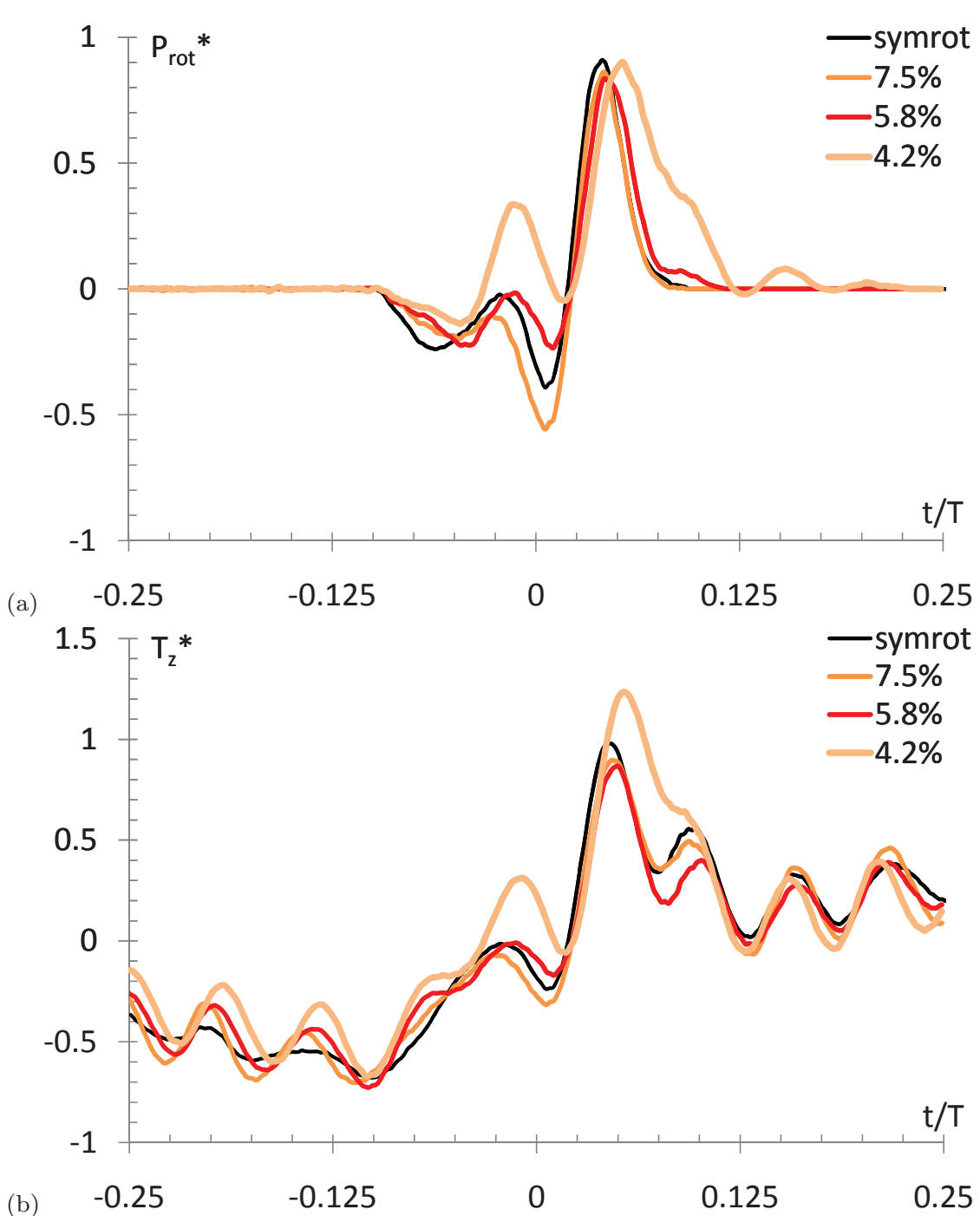

Fig. 13 Dimensionless rotational power, $P_{\text {rot }}^{*}(\mathrm{a})$, and torque, $T_{z}^{*}$ (b). For (a) and (b) the values are plotted during half a period, for the standard symmetrical rotation, symrot, and selected values of $\Delta t$, i.e. $0.075 \mathrm{~T}, 0.058 \mathrm{~T}, 0.042 \mathrm{~T}$, with $r_{k p}=0.54 c$. These plots correspond to a phase average over periods 10 to $19 . \mathrm{t} / \mathrm{T}$ is plotted in abscissa. 


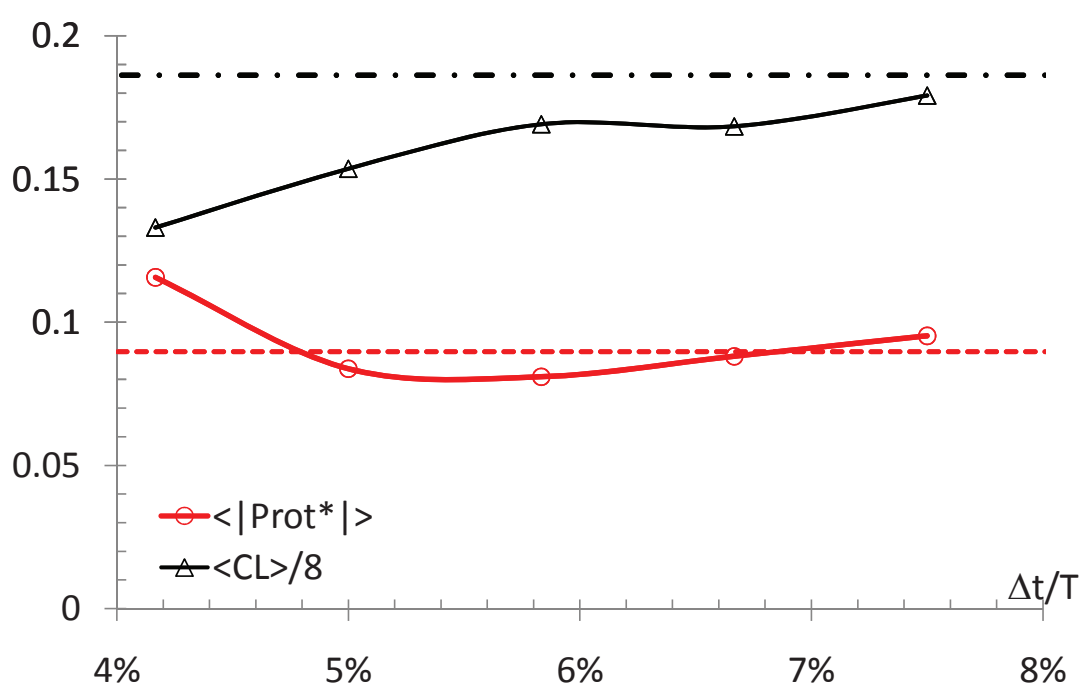

Fig. 14 Mean non-ideal rotational power, $\left\langle\left|P_{\text {rot }}^{*}\right|\right\rangle$, and mean lift coefficient, $\left\langle C_{L}\right\rangle / 8$, versus $\Delta t$ values given in abscissa in $\%$ of $\mathrm{T}$ with $r_{k p}=0.54 c$. The dot-dash and dashed lines indicate the values obtained for the symmetrical rotation for lift and power respectively. 


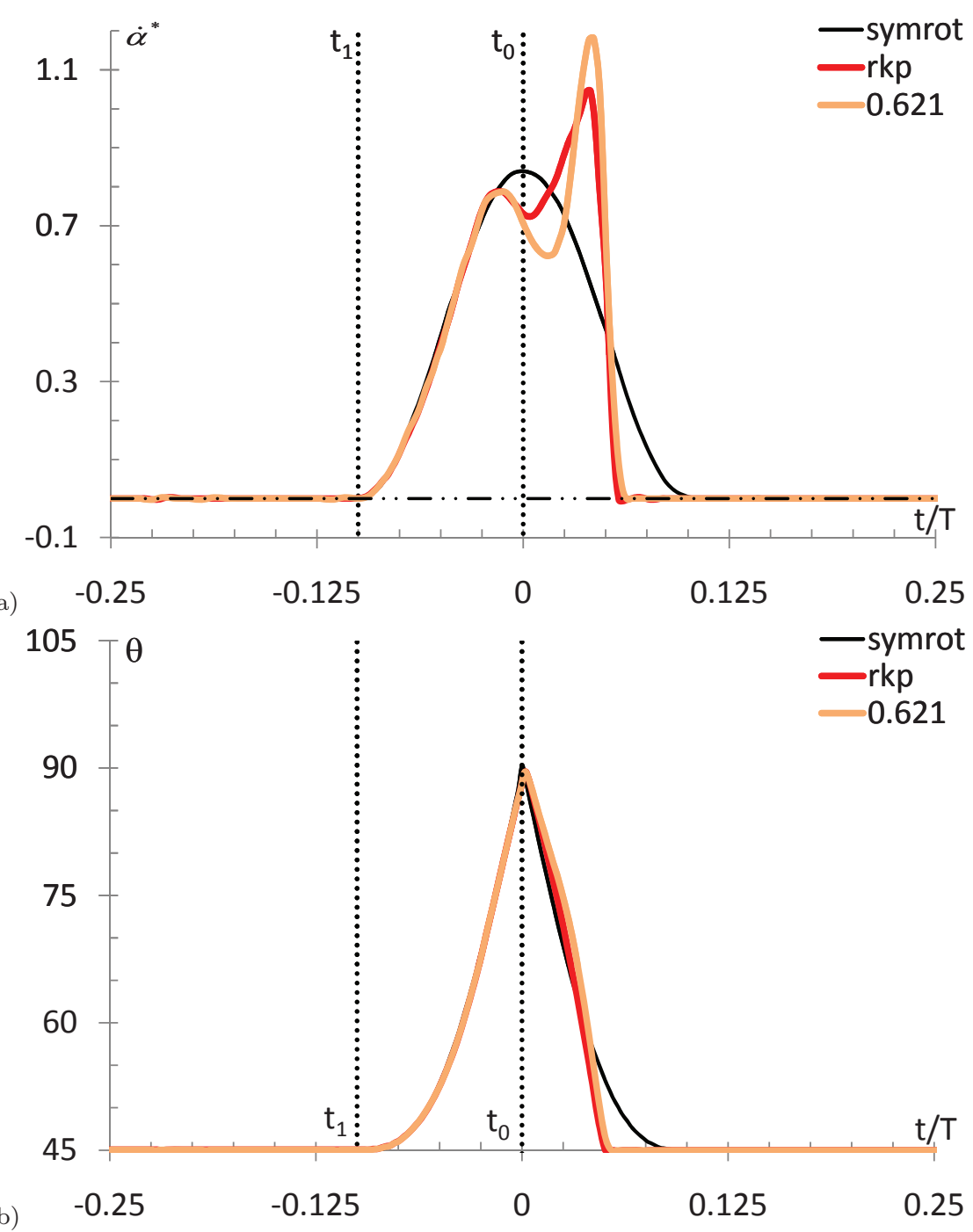

Fig. 15 Dimensionless angular velocity, $\dot{\alpha}^{*}(\mathrm{a})$, and geometrical angle, $\theta$ (b), of the wing. For (a) \& (b) the values are plotted during half a period, for the standard rotation, noted symrot, the natural double bump kinematic, noted rkp, and a tuned double bump noted 0.621 for $p=0.621$. The curves plotted are exported from recorded experimental data. $\mathrm{t} / \mathrm{T}$ is plotted in abscissa. On (a), the dash-dot-dot horizontal line indicates $\dot{\alpha}^{*}=0$. 

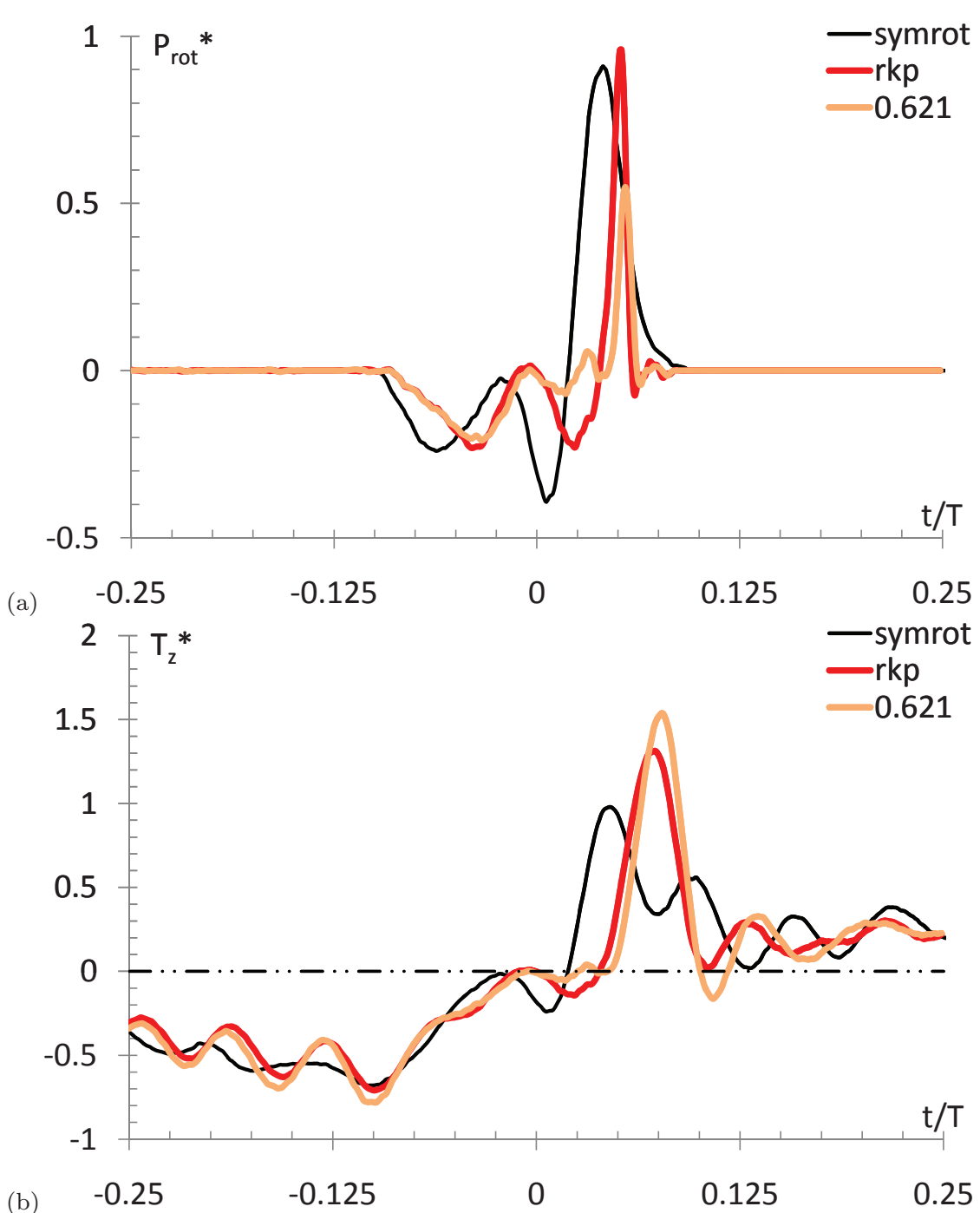

Fig. 16 Dimensionless rotational power, $P_{\text {rot }}^{*}$ (a), and torque, $T_{z}^{*}$ (b). For (a) \& (b) the values are plotted during half a period for the standard rotation, noted symrot, the natural double bump kinematic, noted rkp, and a tuned double bump noted 0.621 for $p=0.621$. These power plots correspond to a phase average over periods 10 to 19. t/ T is plotted in abscissa. On (b), the dash-dot-dot horizontal line indicates $T_{z}^{*}=0$ 


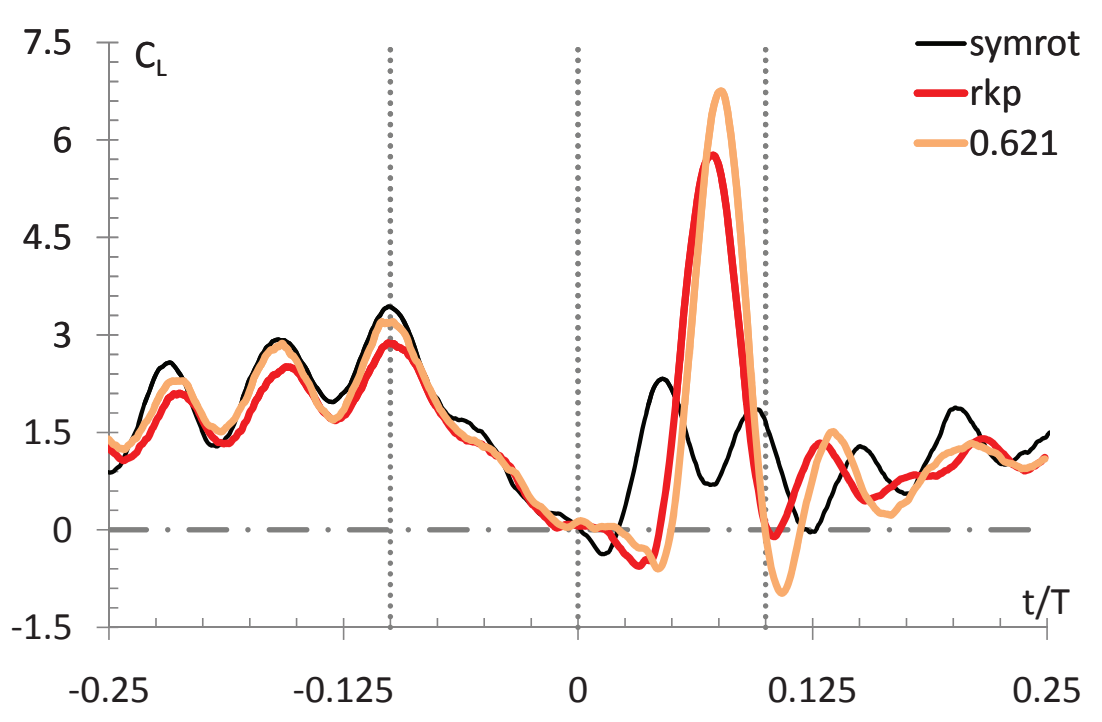

Fig. 17 Lift coefficient, $C_{L}$ versus t/T. The values are plotted during half a period for the standard rotation, noted symrot, the natural double bump kinematic, noted rkp, and a tuned double bump noted 0.621 for $p=0.621$. These values correspond to a phase average over periods 10 to 19 .

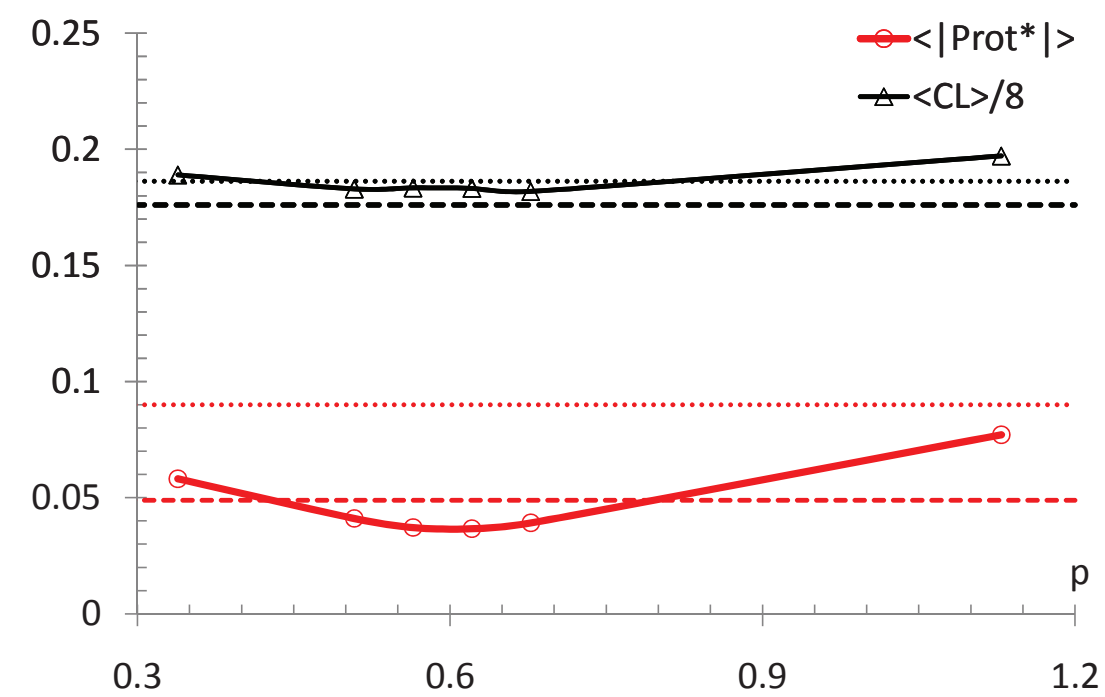

Fig. 18 Average, over 10 flapping (period 10 to 19), of the non-ideal rotational power, $<\left|P_{r o t}^{*}\right|>$, and the lift coefficient, $\left\langle C_{L}\right\rangle / 8$, versus p values given in abscissa. Dashed horizontal lines correspond to the values obtained for the natural double bump, previously noted rkp in Fig. 15 and 13. The dotted horizontal lines correspond to the values obtained for the symmetrical rotation. 